\title{
Prediction of Ultrafine Particle Number Concentrations in Urban Environments by means of Gaussian Process Regression Based on Measurements of Oxides of Nitrogen
}

\author{
Matteo Reggente ${ }^{\mathrm{a}, *}$, Jan Peters ${ }^{\mathrm{a}}$, Jan Theunis ${ }^{\mathrm{a}}$, Martine Van Poppel ${ }^{\mathrm{a}}$, \\ Michael Rademaker ${ }^{\mathrm{b}}$, Prashant Kumar ${ }^{\mathrm{c}, \mathrm{d}}$, Bernard De Baets ${ }^{\mathrm{b}}$ \\ ${ }^{a}$ VITO, Flemish Institute for Technological Research, Boeretang 200, B-2400 Mol, \\ Belgium \\ ${ }^{b}$ Department of Mathematical Modelling, Statistics and Bioinformatics, Ghent \\ University, Coupure links 653, 9000 Ghent, Belgium \\ ${ }^{c}$ Department of Civil and Environmental Engineering, Faculty of Engineering and \\ Physical Science (FEPS), University of Surrey, GU2 7XH, United Kingdom \\ ${ }^{d}$ Environmental Flow (EnFlo) Research Centre, FEPS, University of Surrey, GU2 7XH, \\ United Kingdom
}

\begin{abstract}
Gaussian process regression is used to predict ultrafine particle (UFP) number concentrations. We infer their number concentrations based on the concentrations of $\mathrm{NO}, \mathrm{NO}_{2}, \mathrm{CO}$ and $\mathrm{O}_{3}$ at half hour and five minutes resolution. Because UFP number concentrations follow from a dynamic process, we have used a non-stationary kernel based on the addition of a linear and a rational quadratic kernel. Simultaneous measurements of UFP and gaseous pollutants were carried out during one month at three sampling locations situated within a $1 \mathrm{~km}^{2}$ area in a Belgian city, Antwerp. The method proposed provides accurate predictions when using $\mathrm{NO}$ and $\mathrm{NO}_{2}$ as covariates and less accurate predictions when using $\mathrm{CO}$ and $\mathrm{O}_{3}$. We have also evaluated the models for different training periods and we have found that a
\end{abstract}

\footnotetext{
${ }^{*}$ Corresponding author

Email address: reggente@gmail.com (Matteo Reggente)
} 
training period of at least seven days is suitable to let the models learn the UFP number concentration dynamics in different typologies of traffic.

Keywords:

Ultrafine particles; Number distributions; Street canyon; Traffic emissions; Gaussian process regression; Urban air pollution

\section{Introduction}

Exposure to ultrafine particles (UFP) is of great concern because of their adverse impacts on human health (Atkinson et al., 2010). UFP are commonly defined as particles having a diameter of less than 100nm (Morawska et al., 1998), and the consensus is that these particles contribute most (around 80\%) to the total particle number concentration (PNC) (Heal et al., 2012; Kumar et al., 2011c; Morawska et al., 2008; Charron and Harrison, 2003), whereas their corresponding mass accounts for less than $20 \%$ of the total particle mass concentration (Kittelson, 1998). UFP can be classified into the "nucleation", "Aitken" and "accumulation" modes. In terms of size ranges, the nucleation, Aitken and accumulation modes typically encompass 1-30, 20-100 and 30-300nm, respectively. Particles with a diameter below $30 \mathrm{~nm}$ contain nearly $30 \%$ of total PNC (Morawska et al., 2008; Kumar et al., 2010).

UFP can cause damage to pulmonary cells, and may penetrate and deposit in the tissues. Kumar et al. (2011a) have shown significant contributions to excess mortality due to the exposure to traffic-derived UFP in urban areas. Jacobs et al. (2010) and Bos et al. (2011) have shown that even brief exposure of healthy people to traffic UFP seems to induce some health effects. Moreover, those particles may also cause cardio-vascular damage 
(de Hartog et al., 2003) and could cause brain stroke when penetrating into the bloodstream and being transported to other organs (Hong et al., 2002).

Road vehicle emissions in polluted urban environments can contribute up to $90 \%$ of the total particle number concentration (PNC) (Kumar et al., 2010; Pey et al., 2009). The UFP number concentrations along the roadside show an association with the vehicle flow characteristics. For instance, increasing vehicle speed increases the emissions of UFP (Kittelson et al., 2004). Among the road vehicles, diesel engines dominate road traffic emission of UFP, and heavy duty vehicles have an average factor of magnitude of two with respect to the light duty engine (Beddows and Harrison, 2008).

Nitrogen dioxide $\left(\mathrm{NO}_{2}\right)$ is a well-known traffic-related pollutant. Together with nitric oxide (NO), they are known as nitrogen oxides (NOx). NOx emissions are associated with all types of high-temperature combustion, but similarly to the UFP, the most important source in urban areas are the vehicles (Westmoreland et al., 2007). Direct NOx emissions from vehicles are mainly emitted as NO (Soltic and Weilenmann, 2003), and they quickly react with ozone $\left(\mathrm{O}_{3}\right)$ resulting in secondary $\mathrm{NO}_{2}$ formation (Westmoreland et al., 2007). The $\mathrm{NO}_{2}$ share of emitted NOx is increasing (Keuken et al., 2009) due to the increased number of diesel vehicles, especially those equipped with exhaust after-treatment systems (i.e. CRT - continuous regenerating trap) which use $\mathrm{NO}_{2}$ to regenerate the filter: soot particles are burned at $550^{\circ} \mathrm{K}$ using $\mathrm{NO}_{2}$ (Alvarez et al., 2008).

UFP vary spatially between the sources and the humans living or travelling close to the roads. This variation depends on many factors such as source type and strength, meteorological and dilution conditions, location geometry and transformation processes, among others (Heal et al., 2012). For example, studies carried out near highways show that number concen- 
trations of particles decrease exponentially with the increasing downwind distance from the highway (Buonanno et al., 2009; Fujitani et al., 2012). Such a decrease was observed up to $\sim 300 \mathrm{~m}$ and beyond, at which point levels of UFP number concentrations approached the local urban background (Morawska et al., 1999; Zhu et al., 2002).

In urban environments, the UFP variability has been studied by Kumar et al. (2009), showing that typically their number concentration close to the exhaust pipe is in the order of $\sim 10^{6}-10^{7} \mathrm{~cm}^{-3}$ and two to three orders of magnitude less on the roadside, $\sim 10^{4}-10^{5} \mathrm{~cm}^{-3}$. The same work also investigated the temporal variability of the UFP using a fast response $(10 \mathrm{~Hz})$ measurement device. They found that the transformation processes in the nucleation mode are nearly complete within about one second after the emission, and considering that the emissions can take tens of second to reach the road side, explaining thus the three order magnitude concentration differences found close to the emission and on the road side. Wehner et al. (2009) reported that number concentrations measured behind a diesel car are up to two orders of magnitude above the roadside concentrations. Kumar et al. (2008) extended the characterization of UFP concentrations studying their vertical variation. They found that UFP number concentration at a height of $2.6 \mathrm{~m}$ were up to $40 \%$ lower than those at ground level.

Currently there is no limit value to control the number concentration of UFP. At the European level, a standard for number concentrations emissions of vehicles "is to be defined as soon as possible and at the latest upon entry into force of Euro 6" (Regulation (EC) No 715/2007). Moreover, air quality (AQ) standards focus on mass concentrations of PM (PM2.5; PM10) and no AQ limit values exist for particle number concentrations. Consequently, there are currently not many UFP monitors deployed in the governmen- 
tal monitoring stations. On the other hand, $\mathrm{NO}_{2}$ is a regulated pollutant (Directive 2008/50/EC) and monitors are spread all over Europe. In this paper we use statistical modelling to estimate the UFP number concentration using the information content of the $\mathrm{NO}, \mathrm{NO}_{2}, \mathrm{CO}$ and $\mathrm{O}_{3}$ readings.

UFP number concentration is itself a response variable dependent on covariates such as atmospheric conditions, local meteorology and other spatial and temporal factors. Its response is thus site and time specific (Kulmala et al., 2004) and the transformation processes that change the UFP number and size distributions differ both temporally and spatially (Kumar et al., 2011b), making the modelling of this pollutant a really challenging task (Nikolova et al., 2011).

The modelling of pollutants mostly fits into two categories: deterministic dispersion models and stochastic prediction models. Deterministic dispersion models provide a link between theory and measurements (Mølgaard et al., 2012) and account for source dynamics and physico-chemical processes (e.g. transport mechanisms, chemical reactions) explicitly. As a drawback, these models need detailed information which is not always available. Holmes and Morawska (2006) reviewed different particle dispersion models and concluded that many factors influence the concentration of UFP, notably fluctuations in the wind flow and emissions.

Stochastic prediction models do not describe the actual physical processes, but they treat the input data as random variables and derive a statistical description of the target distribution using a set of measurements to learn the expected pollutant concentration. Statistical approaches offer complementary strengths compared to the deterministic dispersion models. These models do not rely on the validity of the underlying physical model, but can provide a higher resolution and are computationally less expensive 
and generally less demanding in terms of the required knowledge about the state of the environment.

In this paper we treat the prediction of the UFP number concentration in the Aitken and accumulation modes (size range $25-300 \mathrm{~nm}$ ) as a supervised learning regression problem. In other words, the model tries to learn the similarities existing between its inputs (covariates: $\mathrm{NO}, \mathrm{NO}_{2}, \mathrm{CO}$ and $\mathrm{O}_{3}$ ) and its target (UFP). More precisely, a Gaussian process (GP) regression model is used (Rasmussen and Williams, 2006). This model has been favourably compared to back-propagation neural networks (NN) and other models (Rasmussen, 1996). This work focuses on UFP with size larger than $25 \mathrm{~nm}$. Smaller particles in the nucleation mode are volatile and unstable in nature, and it is challenging to accurately measure them (Morawska et al., 2008). This is also one of the reasons why particles below $\sim 25 \mathrm{~nm}$ are not part of current Euro 5/6 vehicle emission standards. Limiting the study to this size range takes out the uncertainty involved in measuring nucleation mode particles with relatively shorter atmospheric life time. The same criterion has been adopted in Mishra et al. (2012).

The article is structured as follows. In Section 2, a literature review on statistical modelling of UFP in relation to this work is provided. Sections 3 and 4 give a description of the sites and the instrumentation used, and the UFP prediction model, respectively. Section 5 details the model evaluation metrics we use in this paper. Section 6 presents the extensive evaluation of the model and investigates its performance on varying training data volumes. 


\section{Related work in statistical UFP modelling}

Mølgaard et al. (2012) use Bayesian supervised regression to predict up to two days ahead size-fractionated UFP number concentrations of an urban background monitor station in Helsinki. They use meteorological and traffic data as inputs to their regression model (covariates) and as target the size-fractioned PNC provided by a particle monitor located at the urban background station. The inputs of the prediction model are the actual values of the covariates. They compare the output of their model with the value measured by the monitoring station reporting an $\mathrm{R}^{2}$ of 0.67 at a three hours resolution. The data from the same monitoring station (2004-2006) have been used (Clifford et al., 2011) to model the UFP number concentrations using a generalized additive model and meteorological data, time, solar radiation and rainfall as covariates, reporting an $\mathrm{R}^{2}$ of 0.836 for their fitted model. Hussein et al. (2006) use the data from a background monitoring station. They found that the covariates that contained the most information are the ambient temperature and local wind conditions. They fit a linear regression model after dividing the entire dataset in wind sectors of $10^{\circ}$. None of the mentioned works uses regulated pollutants as covariates to predict UFP number concentrations.

Sabaliauskas et al. (2012) predict UFP number concentrations in the 8-50nm $\left(\mathrm{PN}_{50}\right)$ and 50-100nm $\left(\mathrm{PN}_{50-100}\right)$ size ranges using NOx and meteorological data in a multiple linear regression model, based on a roadside five years long measurement campaign. Their model explains $52 \%\left(\mathrm{R}^{2}=0.52\right)$ of the variance in the case of $\mathrm{PN}_{50}$ and $63 \%\left(\mathrm{R}^{2}=0.63\right)$ in the case of $\mathrm{PN}_{50-100}$. It should be noted that NOx and UFP were not measured at the same site, but at a distance of $750 \mathrm{~m}$ from each other. Considering the local character- 
istic of UFP (especially for the freshly emitted nucleation mode particles), this could explain the low performance of their regression model in capturing the day-to-day variability. This is also supported in the same study, in which they were able to predict bigger particles (in the range 100-300nm) explaining $83 \%$ of the variance. It is worth noting that none of these works mentions the length of the training period, nor investigates its effect on the model performance.

From this short description it is clear that there still is a lack of models for predicting UFP number concentration based on variables that are currently being measured in air quality monitoring networks. The novelty of our work lies in the following:

- As opposed to the previous works (Mølgaard et al., 2012; Clifford et al., 2011; Hussein et al., 2006; Sabaliauskas et al., 2012), we have used simultaneous measurements of $\mathrm{NO}, \mathrm{NO}_{2}, \mathrm{CO}$ and $\mathrm{O}_{3}$ (covariates of the model) and UFP number concentrations (target variable) at three different locations, obtaining a similar model performance at all three locations.

- Compared to the work presented by Mølgaard et al. (2012), Clifford et al. (2011) and Hussein et al. (2006), we have used NO, $\mathrm{NO}_{2}, \mathrm{CO}$ and $\mathrm{O}_{3}$ as covariates. These data are usually available from the governmental monitoring stations in urban environments. Unlike the study of Sabaliauskas et al. (2012), we have measured UFP number concentrations and NOx at the same location.

- The previous works (Mølgaard et al., 2012; Clifford et al., 2011; Hussein et al., 2006) have used data from an urban background, while the 
data from our study were gathered at or near the roadside where the variability of the UFP is likely to be higher.

- We have studied the change in prediction performance when changing the amount of data used for training.

- The temporal resolution at which predictions are made in this study (half hour and five minutes) is higher than in most of the studies reported in the literature.

\section{Material}

\subsection{Description of the sampling locations}

Measurements were carried out in the Borgerhout district $\left(51^{\circ} 13^{\prime} \mathrm{N}\right.$ and $4^{\circ} 26^{\prime} \mathrm{E}$ ) of Antwerp, Belgium, which is a port city of approximately 500, 000 inhabitants. Borgerhout has a surface area of $3.93 \mathrm{~km}^{2}$ and houses about 44, 000 inhabitants (as of April $4^{\text {th }}$ 2011). It is a typical urban commercial and residential area with busy traffic. Measurement campaigns were carried out simultaneously for one month (12 ${ }^{\text {th }}$ February until $12^{\text {th }}$ March) during the winter of 2010 at three different sites (described in Table 1). The sites were within an area of about $1 \mathrm{~km}^{2}$. Specific differences between sites (e.g. exact distance to the axis of the road) and meteorological conditions (low dispersion conditions during winter) may be reflected in the UFP measurements. The location of the sampling sites is shown in Figure 1.

Site 1 was located in a street canyon that is close to an open space and has a north-south orientation. The road has two traffic lanes and moderate levels of traffic. Here the monitoring devices were deployed in a parking lot $(\sim 3 \mathrm{~m}$ away from the traffic). 
Site 2 was located in a two lane street canyon. The site experiences moderate traffic, including two tram lines. The road has a northwest-southeast orientation. The sampling location was at the southern corner of the street $(\sim 2 \mathrm{~m}$ away from the traffic).

Site 3 was located in a parking area $\sim 20-30 \mathrm{~m}$ away from a major access road to the city with an east-west orientation, with busy traffic intersections and four lanes (two in each direction) and $\sim 200 \mathrm{~m}$ away from a highway (the ring of the city). Further details on the traffic at each site are presented in Table 1.

Table 1: Description of the measurement sites.

\begin{tabular}{lllll}
\hline & $\begin{array}{l}\text { Distance from } \\
\text { traffic }(\mathrm{m})\end{array}$ & $\begin{array}{l}\text { Weekday } \\
\text { traffic volume } \\
(\text { veh/day) }\end{array}$ & $\begin{array}{l}\text { Weekend } \\
\text { traffic volume } \\
(\text { veh/day) }\end{array}$ & $\begin{array}{l}\text { Heavy weekday } \\
\text { on }\end{array}$ \\
\hline Site 1 & $\sim 3$ & 5000 & 4000 & $5 \%(2 \%)$ \\
Site 2 & $\sim 2$ & 4000 & 3000 & $4 \%(2 \%)$ \\
Site 3 & $\sim 20-30$ & 37000 & 25000 & $7 \%(3 \%)$ \\
\hline
\end{tabular}

\subsection{Instrumentation}

Measurements of UFP were obtained using the GRIMM Nano-Check model 1.320 (Grimm), which is operated with a Grimm 1.108 optical aerosol spectrometer in an environmental weather housing model 1.165. The NanoCheck uses a diffusion charger and Faraday cup electrometer. The mean particle diameter is determined by varying (high and low) the voltage through the electrometer. Comparison of the two resulting currents versus a factory calibration curve gives the mean particle diameter. The Nano-Checks were installed at each of the three sites for simultaneous measurements, enabling 


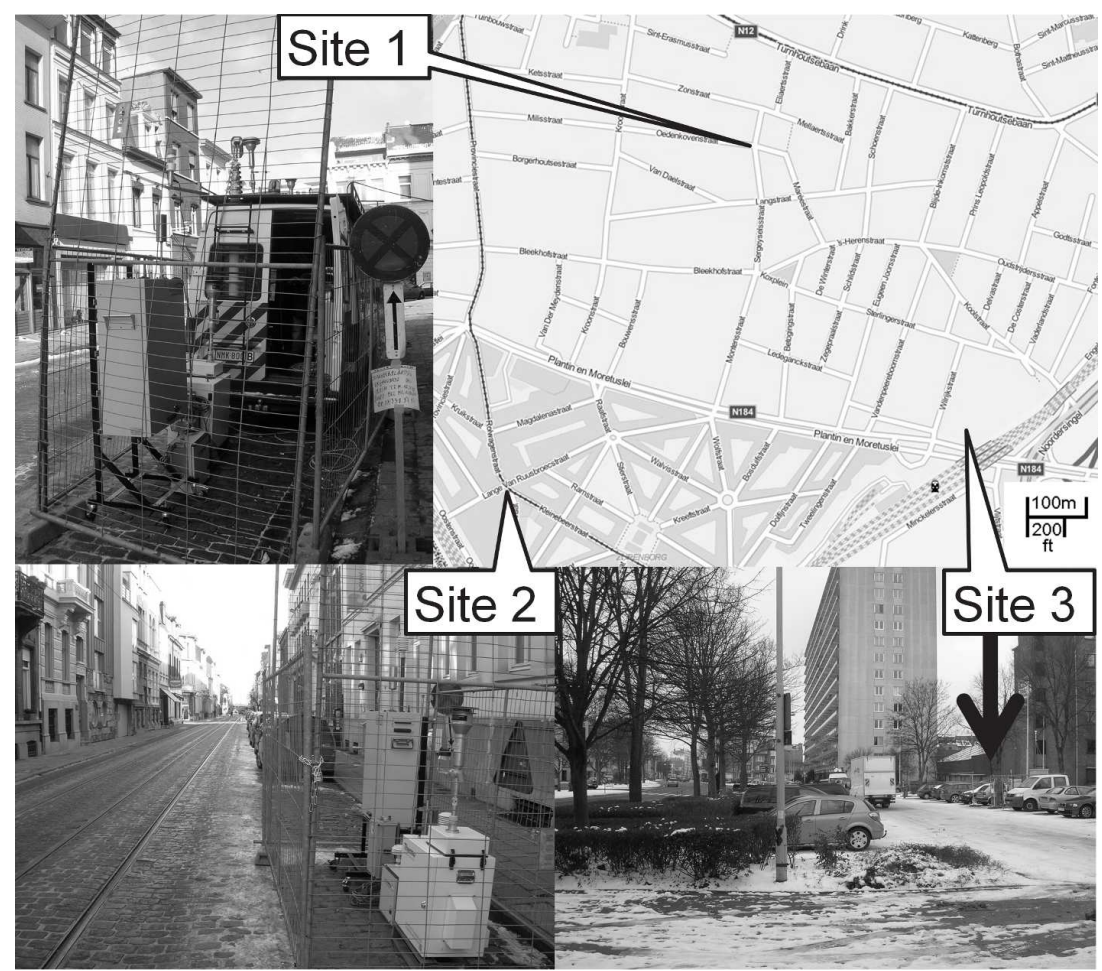

Figure 1: Map of the measurement locations in Borgerhout (Antwerp, Belgium). The photos show the deployed instrumentation at each site. The black arrow in the photo of site 3 shows the location of the deployed monitors. 
a temporal comparison of UFP concentrations measured by identical instruments. Each instrument was calibrated by Grimm using $\mathrm{NaCl}$ particles (size range 27.8-177). The Nano-Check can count total PNCs between 25-300nm, besides providing the mean diameter of the measured size range. The sampling frequency was five minutes and the sampling inlet of the instruments was about $1.6 \mathrm{~m}$ above ground level. Using a threshold of $25 \mathrm{~nm}$ reduced the uncertainty involved in measuring volatile and unstable nucleation mode particles that have a relatively short atmospheric life time (Morawska et al., 2008). The deployed instruments are thus fit for long term representative measurement of UFP.

Chemiluminescence (EN 14211), ultraviolet photometry (EN 14625) and non-dispersive infrared (EN14626) based analysers (Air-pointer, Recordum Austria) were used to measure $\mathrm{NO}, \mathrm{NO}_{2}, \mathrm{CO}$ and $\mathrm{O}_{3}$, respectively. The lowest detectable concentration was $1 \mu \mathrm{g} \mathrm{m} \mathrm{m}^{-3}$ for $\mathrm{NO}, \mathrm{NO}_{2}$ and $\mathrm{O}_{3}$, and $50 \mu \mathrm{g} \mathrm{m}^{-3}$ for CO. The inlet of the gas analysers was at $2 \mathrm{~m}$ above the ground level. We recorded UFP number concentrations and gaseous pollutants for one month at a sampling frequency of five minutes and then averaged on a half hourly basis.

Vehicle counts and speeds were recorded in four vehicle categories (cars, vans, small and big trucks/buses) using double inductive loop detectors at sites 1 and 3 ; video counting was performed to obtain traffic data at site 2 .

The UFP data set used in this work was previously used and discussed in (Mishra et al., 2012).

\subsection{Summary statistics}

Figure 2 (and Table A.4 in the supplementary material section) provides the summary statistics for UFP, $\mathrm{NO}, \mathrm{NO}_{2}, \mathrm{CO}$ and $\mathrm{O}_{3}$ concentrations 
recorded over the entire sampling period. The UFP concentrations (according to the medians and the inter-quartile ranges) are similar at all three sites, although traffic density (Table 1 ) at site 3 is almost one order of magnitude higher than the traffic densities at the other two sites. This indicates that the traffic volume is not the only decisive factor in the variations of the UFP number concentrations at the chosen sites, but also the distance from the moving traffic (a few meters for sites 1 and 2, and 20-30m for site 3). This observation shows a similar trend to those reported in literature (e.g., Buonanno et al., 2009), where the UFP number concentrations were found to decrease by nearly $50 \%$ compared to the roadside values at about $30 \mathrm{~m}$ away from the highway. These observations are also in agreement with the study of Kumar et al. (2009) and Fujitani et al. (2012), especially given that the short lived nucleation particles are not monitored in this study.

Similarly, the $\mathrm{NO}, \mathrm{NO}_{2}, \mathrm{CO}$ and $\mathrm{O}_{3}$ concentrations (according to the medians and the inter-quartile ranges) are similar at all three sites. The higher traffic intensity at site 3 as compared to sites 1 and 2, is again probably offset by the larger distance to the traffic (Table 1) and resulting pollutant dilution.

\subsection{Log-linear regression analysis}

Road vehicles in polluted urban environments can contribute up to $90 \%$ of total particle number concentrations (Kumar et al., 2010; Pey et al., 2009). Figure 3 reports the log-linear regression line (and $95 \% \mathrm{CI}$ ), $\mathrm{R}^{2}$ and RMSE between the UFP number concentrations at each site and the concentrations of $\mathrm{NO}, \mathrm{NO}_{2}, \mathrm{CO}$ and $\mathrm{O}_{3}$ measured at the same site. $\mathrm{NO}$ and $\mathrm{NO}_{2}$ have a strong correlation with UFPs (showing $\mathrm{R}^{2}$ values between 0.6 and 0.74 for $\mathrm{NO}$, and roughly 0.8 for $\mathrm{NO}_{2}$ ). Correlation with $\mathrm{CO}$ is clearly 

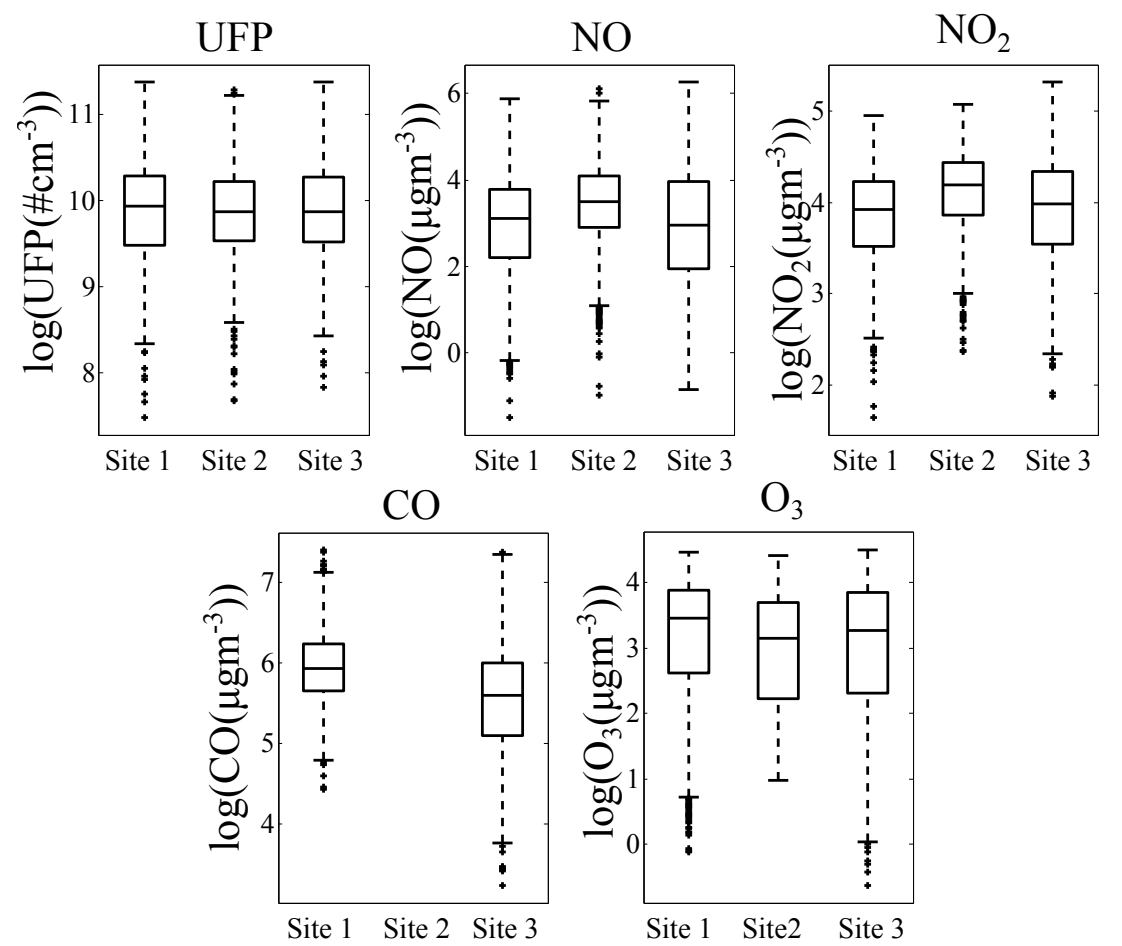

Figure 2: Summary statistics of UFP number concentrations, $\mathrm{NO}, \mathrm{NO}_{2}, \mathrm{CO}$ and $\mathrm{O}_{3}$ data, measured at the three sites. 
lower $\left(\mathrm{R}^{2} \approx 0.4\right.$ to 0.5$)$. Several authors reported correlations between UFP, NOx and CO (e.g., Janhäll et al., 2004; Sardar et al., 2005; Westerdahl et al., 2005; Hagler et al., 2010), although in varying degrees depending on lower UFP cut-off diameter, distance to traffic, traffic composition and season. Morawska et al. (2008) conclude that while in general there is a reasonably good correlation between UFP and traffic emitted gaseous pollutants, the existence and the degree of correlation varies. In our case correlations with $\mathrm{NO}_{2}$ and $\mathrm{NO}$ are higher than with $\mathrm{CO}$, probably because the car fleet in Belgium has a large share of diesel vehicles (Beckx et al., 2013), which have an emission profile that is high in both UFP and NOx (Beddows and Harrison, 2008). The UFP number concentrations have a weak negative correlation with $\mathrm{O}_{3}$. This is in line with findings from (Sardar et al., 2005) who find weak negative correlations with ozone in fall and winter.

\section{Description of the model}

\subsection{Bayesian modelling}

The principle of Bayesian inference is to construct the posterior probability distribution for the unknown entities in a model $(\mathcal{M})$ given the data sample $(\mathcal{D})$. The model $\mathcal{M}$ includes the hypotheses and assumptions made (e.g. covariance function of the Gaussian process; residual model; covariates) and the results are conditioned on $\mathcal{M}$.

The posterior probability for the parameters $(\theta)$ contains all the information about $\theta$ that we are able to extract from the data $(\mathcal{D})$ with the model $(\mathcal{M})$ we are using. According to Bayes rule

$$
p(\theta \mid \mathcal{D}, \mathcal{M})=\frac{p(\mathcal{D} \mid \theta, \mathcal{M}) p(\theta \mid \mathcal{M})}{p(\mathcal{D} \mid \mathcal{M})}
$$




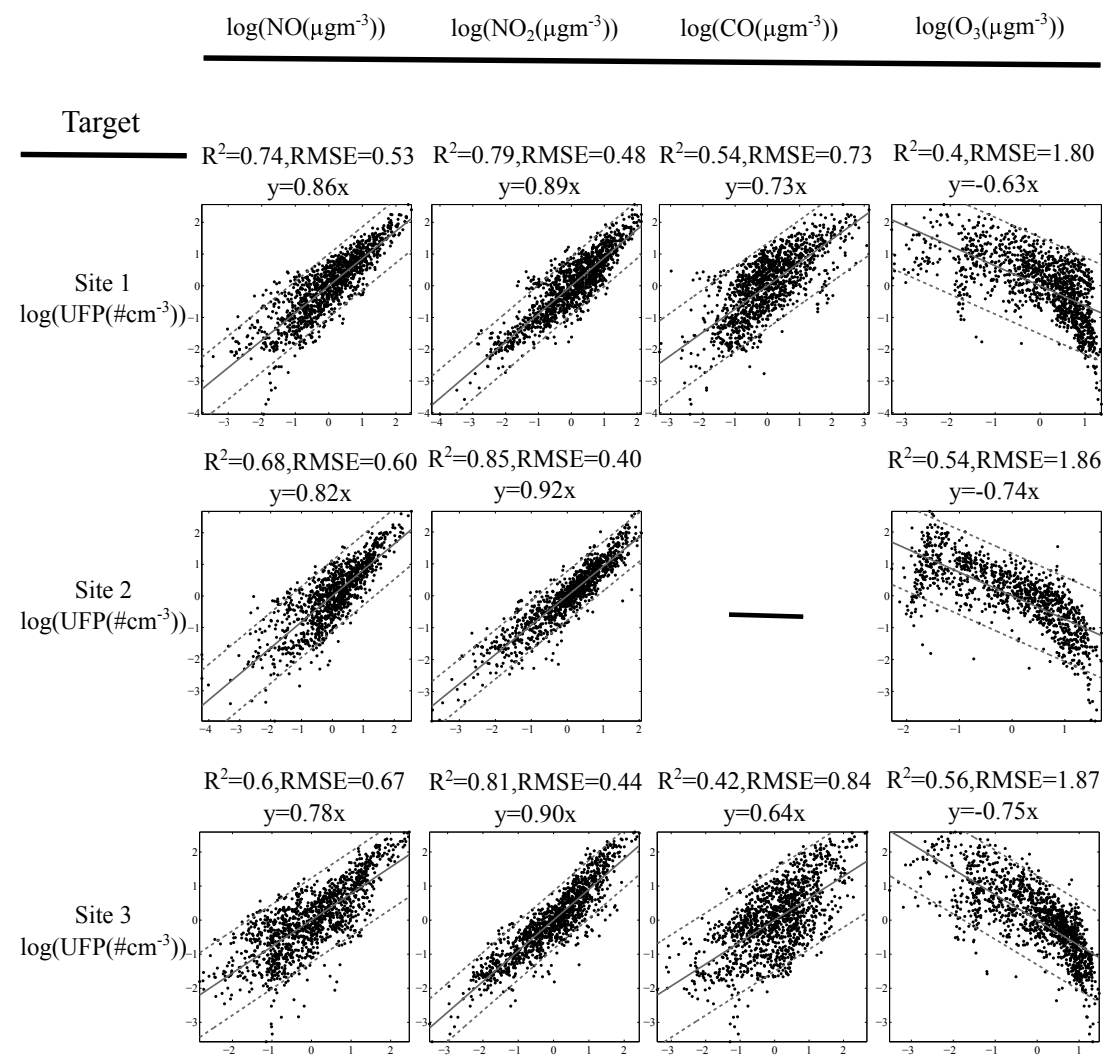

Figure 3: Log-linear regression between standardised UFP and local NO (first column), $\mathrm{NO}_{2}$ (second column), $\mathrm{CO}$ (third column) and $\mathrm{O}_{3}$ (fourth column). Each scatterplot shows the regression line (gray lines), 95\% CI (dashed gray lines), $\mathrm{R}^{2}$ and RMSE. Top row refers to Site 1, middle row refers to Site 2 and bottom row refers to Site 3. 
where, where $p(\mathcal{D} \mid \theta, \mathcal{M})$ is the likelihood of the parameters $\theta, p(\theta \mid \mathcal{M})$ is the prior probability of $\theta$, and $p(\mathcal{D} \mid \mathcal{M})$ is the marginal likelihood, which is independent of the parameters $(\theta)$ and is given by

$$
p(\mathcal{D} \mid \mathcal{M})=\int(\mathcal{D} \mid \theta, \mathcal{M}) p(\theta \mid \mathcal{M}) d \theta
$$

As in Eqs. (1) and (2) everything is conditioned on $\mathcal{M}$, it is a redundant symbol and is as such omitted from here on. Moreover, the model used in this work is not parametric and $\theta$ is replaced by a latent function $f(\mathbf{x})$.

\subsection{Gaussian process regression}

We treat the prediction problem as a non-parametric regression problem, and solve it using GP regression (Rasmussen and Williams, 2006). We do not assume a parametric form of the underlying function $f(\cdot)$ which relates the target variable to the covariates in the form

$$
y=f(\mathbf{x})+\epsilon
$$

In this way, GP regression overcomes one of the major drawbacks of parametric regression, where the functional form $f(\cdot)$ of the relation between covariates and target variable must be stated a priori. In other words, we want to learn, from a set of measurements $(\mathcal{D})$, a function (with credibility intervals) $f(\cdot)$ of the relationship existing between the set of covariates $\mathbf{x}$ $\left(\mathrm{NO}, \mathrm{NO}_{2}, \mathrm{CO}\right.$ and $\mathrm{O}_{3}$ ) and the target variable, UFP number concentrations $(\mathbf{y})$. Moreover, we assume that the observed data $\mathbf{y}$ is generated with Gaussian white noise $\left(\epsilon=\mathcal{N}\left(0, \sigma_{n}^{2}\right)\right)$ around the underlying function $f$.

Definition: A Gaussian process is a collection of random variables, any finite number of which have a joint Gaussian distribution (Rasmussen and Williams, 2006). 
A GP defines a distribution over functions $p(f)$ (it can be seen as a generalization of the Gaussian probability distribution), which can be used for Bayesian regression and therefore Bayesian inference can be used to make predictions from data:

$$
p(f \mid \mathcal{D})=\frac{p(f) p(\mathcal{D} \mid f)}{p(\mathcal{D})}
$$

where $f$ is the function that we want to learn.

GP regression performs inference directly in the space of functions, starting with a prior distribution over all possible functions and subsequently learning the target function from data samples. Consider a collection of random functions indexed by a continuous variable $f(\mathbf{x})$. In a GP any finite sequence of these random function variables $\mathbf{f}=\left(\mathbf{f}_{\mathbf{1}}, \mathbf{f}_{\mathbf{2}}, \ldots, \mathbf{f}_{\mathbf{n}}\right)$, with corresponding inputs $\left(x_{1}, \ldots, x_{n}\right)$, follows a multivariate Gaussian distribution:

$$
p(\mathbf{f}) \sim \mathcal{N}(\boldsymbol{\mu}, \mathbf{K}), \quad \boldsymbol{\mu} \in \mathbb{R}^{n}, \mathbf{K} \in \mathbb{R}^{n \times n}
$$

The GP prior is fully specified by its mean function $\boldsymbol{\mu}$ and covariance function $\mathbf{K}$. Very often (and in this paper as well), it is assumed that the mean of the GP prior is zero everywhere. At first glance, this could appear restrictive, but in practice it is not because offsets and simple trends can be eliminated before modelling. Since the expectation is a linear operator for any deterministic mean function $m(\mathbf{x})$, the GP over $f^{\prime}(\mathbf{x}):=f(\mathbf{x})-m(\mathbf{x})$ has zero mean. Moreover, it is worth noting that the posterior GP $p(f \mid \mathcal{D})$ that arises from the regression is not necessarily a zero-mean process. What really relates one observation to another is the covariance matrix $\mathbf{K}$, which is built from a covariance function (or kernel) $k\left(\mathbf{x}, \mathbf{x}^{\prime}\right)$. The covariance function and its hyperparameters $\boldsymbol{\theta}$ used in this work are described in Section 4.3.

Once the mean and covariance functions are defined, everything else 
follows from the basic rules of probability theory applied to multivariate Gaussian distributions. The likelihood (noise model) is given by:

$$
p(\mathbf{y} \mid \mathbf{f})=\mathcal{N}\left(\mathbf{f}, \sigma_{n}^{2} \mathbf{I}\right)
$$

where $\mathbf{I}$ is the identity matrix. Integrating over the unobserved function variables $\mathbf{f}$ (using Eqs. (A.1)-(A.4) in the supplementary material section) gives the marginal likelihood (or evidence) that is used to tune the covariance function:

$$
p(\mathbf{y})=\int p(\mathbf{y} \mid \mathbf{f}) p(\mathbf{f}) d \mathbf{f}=\int \mathcal{N}\left(\mathbf{f}, \sigma_{n}^{2} \mathbf{I}\right) \mathcal{N}(\mathbf{0}, \mathbf{K}) d \mathbf{f}=\mathcal{N}\left(\mathbf{0}, \mathbf{K}+\sigma^{2} \mathbf{I}\right)
$$

\subsubsection{Prediction}

Given a training dataset $\mathcal{D}=\left\{\left(\mathbf{x}_{i}, y_{i}\right)_{i=1}^{n}\right\}=(\mathbf{X}, \mathbf{y})$ where $\mathbf{x}_{i} \in \mathbb{R}^{d}$ are the inputs (covariates) and $y_{i} \in \mathbb{R}$ the targets, the goal of the regression model is to predict the target value $y_{*} \in \mathbb{R}$ at a new point $\mathbf{x}_{*}$ :

$$
p\left(\mathbf{y}_{*} \mid \mathbf{x}_{*}, \mathcal{D}\right)=\int p\left(\mathbf{y}_{*} \mid \mathbf{x}_{*}, f, \mathcal{D}\right) p(f \mid \mathcal{D}) d f
$$

In order to make predictions based on data, we consider the joint Gaussian prior of the noisy training observations $\mathbf{y}$ and the test noisy outputs $\mathbf{y}_{*}$ :

$$
\left[\begin{array}{c}
\mathbf{y} \\
\mathbf{y}_{*}
\end{array}\right]=\mathcal{N}\left(\mathbf{0},\left[\begin{array}{cc}
k(\mathbf{X}, \mathbf{X})+\sigma_{n}^{2} \mathbf{I} & k\left(\mathbf{X}, \mathbf{X}_{*}\right) \\
k\left(\mathbf{X}_{*}, \mathbf{X}\right) & k\left(\mathbf{X}_{*}, \mathbf{X}_{*}\right)+\sigma_{n}^{2} \mathbf{I}
\end{array}\right]\right)
$$

where $\mathbf{X}$ and $\mathbf{X}_{*}$ are the matrices of the training and test inputs, respectively. $k(\mathbf{X}, \mathbf{X})$ refers to the covariance matrix built by evaluating the covariance function $k(\cdot, \cdot)$ for all pairs $\left\langle x_{i}, x_{j}\right\rangle$ of all row vectors of $\mathbf{X}$.

The posterior distribution is obtained by conditioning (Eq. (A.5) in the supplementary material section) the prior on the observed training outputs: 


$$
p\left(\mathbf{y}_{*} \mid \mathbf{y}, \mathbf{X}, \mathbf{X}_{*}\right)=\mathcal{N}\left(\boldsymbol{\mu}_{*}, \boldsymbol{\Sigma}_{*}\right)
$$

where

$$
\begin{gathered}
\boldsymbol{\mu}_{*}=k\left(\mathbf{X}_{*}, \mathbf{X}\right)\left[k(\mathbf{X}, \mathbf{X})+\sigma_{n}^{2} \mathbf{I}\right]^{-1} \mathbf{y} \\
\boldsymbol{\Sigma}_{*}=k\left(\mathbf{X}_{*}, \mathbf{X}_{*}\right)+\sigma_{n}^{2} \mathbf{I}-k\left(\mathbf{X}_{*}, \mathbf{X}\right)\left[k(\mathbf{X}, \mathbf{X})+\sigma_{n}^{2} \mathbf{I}\right]^{-1} k\left(\mathbf{X}, \mathbf{X}_{*}\right)
\end{gathered}
$$

are the posterior mean and the posterior variance, respectively.

\subsubsection{Training a Gaussian process}

The reliability of the regression is dependent on how well we select the covariance function and therefore the covariance hyperparameters $\boldsymbol{\theta}$. One of the major advantages of GP regression over other methods is the ability to select covariance hyperparameters from the training data directly, rather than using a scheme such as cross-validation. The parameters are selected by minimising the negative $\log$ marginal likelihood $-\mathcal{L}$ with respect to the covariance hyperparameters $\boldsymbol{\theta}$. Since by assumption the distribution of the data is Gaussian, the log marginal likelihood is:

$$
\mathcal{L}=\log p(\mathbf{y} \mid \mathbf{x}, \boldsymbol{\theta})=-\frac{1}{2} \mathbf{y}^{\top} \mathbf{K}_{y}^{-1} \mathbf{y}-\frac{1}{2} \log \left|\mathbf{K}_{y}\right|-\frac{n}{2} \log 2 \pi,
$$

where $\mathbf{K}_{y}=k(\mathbf{X}, \mathbf{X})+\sigma_{n}^{2} \mathbf{I}$ is the covariance matrix for the noisy targets $\mathbf{y}$. The values of the hyperparameters which optimizes the marginal likelihood are found using its partial derivative

$$
\frac{\partial \mathcal{L}}{\partial \boldsymbol{\theta}}=\frac{1}{2} \operatorname{trace}\left(\mathbf{K}_{y}^{-1} \frac{\partial \mathbf{K}_{y}}{\partial \boldsymbol{\theta}}\right)+\frac{1}{2} \mathbf{y}^{\top} \frac{\partial \mathbf{K}_{y}}{\partial \boldsymbol{\theta}} \mathbf{K}_{y}^{-1} \frac{\partial \mathbf{K}_{y}}{\partial \boldsymbol{\theta}} \mathbf{K}_{y}
$$

in conjunction with a numerical optimization routine based on conjugate gradients (Section 4.4). The corresponding optimization problem is not 
convex, and therefore precautions against the risk of bad local minima should be taken.

For a detailed description of GP models, prediction and training, we refer to Chapters 2 and 5 in (Rasmussen and Williams, 2006).

\subsubsection{Limitations}

The major limitation of GP regression is the computational complexity, since it requires matrix inversion, which has a complexity of $\mathcal{O}\left(n^{3}\right)$, where $n$ is the number of training data points. Different solutions have been proposed to cope with this problem. Higdon (1998) proposed a kernel mixing approach for dimensionality reduction. This approach is based on the fact that a continuous process can be created by convolving a continuous white noise process with a Gaussian density kernel on a spatially sparse grid (at $j$ knot locations) covering the study region (input space). In case matrix inversion is needed, the kernel mixing approach allows to decrease the computational complexity to $\mathcal{O}\left(j^{3}\right)$, with $j \ll n$. Lavine and Lozier (1999) proposed a Bayesian model for ocean temperatures using a Markov random field. They divided the study region in bins and the conditional posterior distribution of the target variable (temperature) at each location depends only on those within the bin area. Snelson and Ghahramani (2006) reduce the training data set in a way that introduces a small loss in the data likelihood, while at the same time minimizing the runtime (FITC approximation). Rasmussen and Ghahramani (2002) use an infinite mixture of GP, dividing the dataset in clusters and then modelling each of them. Moreover their model allows the effective covariance function to vary with the inputs. In this work we have used the FITC approximation for the five minute resolution model and we have compared it to the full GP model at half hour resolution. 


\subsection{Covariance function}

GP models, and more in general kernel-based nonparametric models, depend on defining a kernel function $k\left(\mathbf{x}, \mathbf{x}^{\prime}\right)$ (covariance) that determines how correlated outputs $y$ and $y^{\prime}$ are expected to be at two inputs $\mathbf{x}$ and $\mathbf{x}^{\prime}$. GP is gaining interest in many application fields and the de facto standard choice for $\left(x, x^{\prime}\right)$ is the squared exponential covariance function $k_{S E}$. Nevertheless, this covariance function is not the proper choice for all applications, as it relies on the basic assumption that it is a function only of $\left\|\mathbf{x}-\mathbf{x}^{\prime}\right\|$ (it is invariant under both translation and rotation, isotropy).

In Figure 4, we have plotted the standardised UFP number concentrations (logarithmic scale) against the standardised inputs $\mathrm{NO}, \mathrm{NO}_{2}, \mathrm{CO}$ and $\mathrm{O}_{3}$ (logarithmic scale). All the plots show that there is a linear trend between the output and inputs of the model. These plots show that the UFP concentrations are increasing (almost linearly) in an unbounded manner in the input space and indicate that the process is not stationary, and therefore, the squared exponential covariance function $k_{S E}$ is not the right choice in the context of this paper.

\subsubsection{Kernel addition}

For the choice of the kernel, we have used the property that positive semidefinite kernels (base kernels) are closed under addition and product (Chapter 4 in (Rasmussen and Williams, 2006)). This allows us to combine base kernels that take into consideration different properties of the process:

$$
k\left(\mathbf{x}, \mathbf{x}^{\prime}\right)=k_{1}\left(\mathbf{x}, \mathbf{x}^{\prime}\right)+k_{2}\left(\mathbf{x}, \mathbf{x}^{\prime}\right)
$$

The sum of two kernels allows us to model the data as a superposition of independent functions representing different structures. 


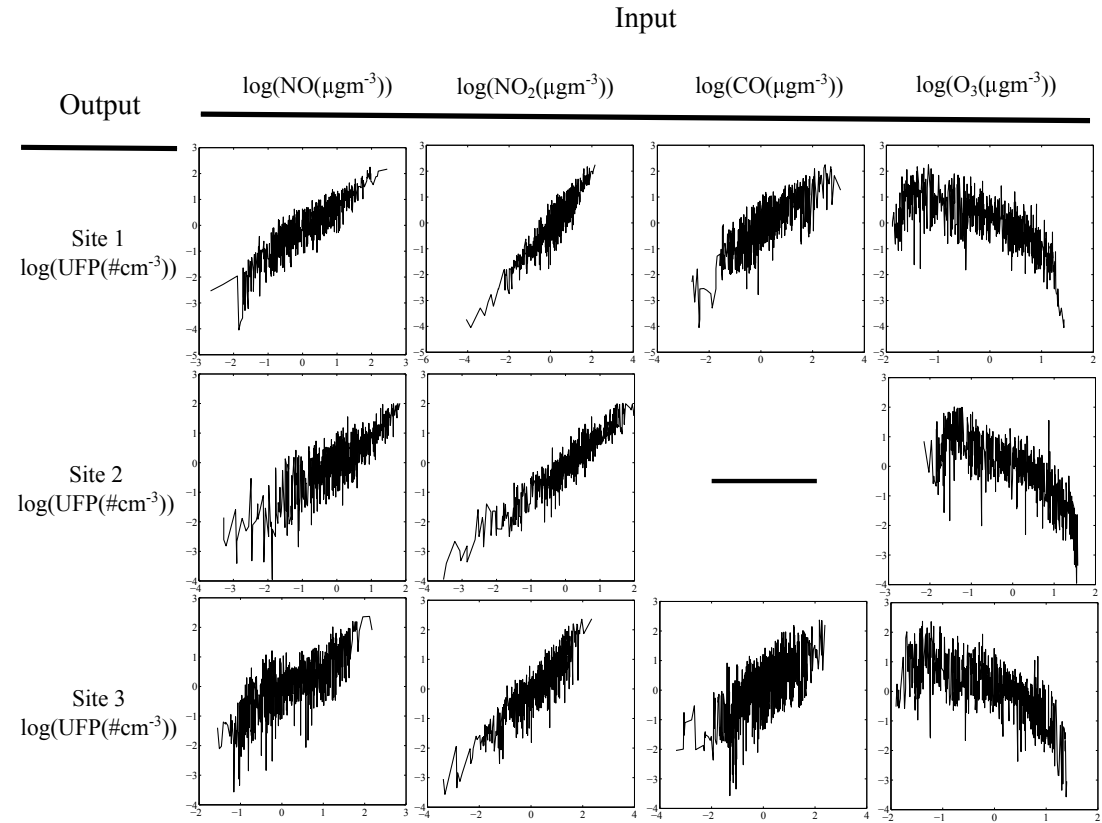

Figure 4: Plots of the UFP against each input feature $\left(\mathrm{NO}, \mathrm{NO}_{2}, \mathrm{CO}\right.$ and $\left.\mathrm{O}_{3}\right)$. 


\subsubsection{Base kernels}

In this work we have used two base kernels: a linear kernel $\left(k_{\text {Lin }}\right)$ and a rational quadratic kernel $\left(k_{R Q}\right)$ :

$$
k_{L i n}\left(\mathbf{x}, \mathbf{x}^{\prime}\right)=\sigma_{0}^{2}+\mathbf{x}^{\top} \mathbf{x}^{\prime}
$$

were the hyperparameter $\sigma_{0}^{2}$ is the offset of the model. The linear kernel is a non-stationary kernel (it neither depends on the relative position of the two inputs nor on their absolute location). The rational quadratic kernel can be seen as a sum of many $k_{S E}$ kernels with different characteristic length-scales:

$$
k_{R Q}\left(\mathbf{x}, \mathbf{x}^{\prime}\right)=\sigma^{2}\left(1+\frac{\left\|\mathbf{x}-\mathbf{x}^{\prime}\right\|^{2}}{2 \alpha \ell^{2}}\right)^{-\alpha}
$$

The hyperparameters are $\sigma$ (magnitude), $\alpha$ (relative weighing) and $\ell$ (lengthscale). GP priors with this kernel expect to see functions which vary smoothly across many length-scales $(\ell)$. The hyperparameter $\alpha$ determines the relative weighing of large-scale and small-scale variations. For $\alpha \rightarrow \infty, k_{R Q}$ is identical to $k_{S E}$. We found that, in this context, the rational quadratic kernel works better than the squared exponential (gives higher marginal likelihood), probably because $k_{R Q}$ can accommodate several length-scales.

\subsubsection{Resulting kernel}

We have used a sum of a linear kernel $\left(k_{\text {Lin }}\right)$ and a rational quadratic kernel $\left(k_{R Q}\right)$ to model the rising trend and the deviations. Inserting Eqs. (16) and (17) in Eq. (15) the resulting kernel is:

$$
k\left(\mathbf{x}, \mathbf{x}^{\prime}\right)=k_{\text {Lin }}\left(\mathbf{x}, \mathbf{x}^{\prime}\right)+k_{R Q}\left(\mathbf{x}, \mathbf{x}^{\prime}\right)
$$

It is possible that other kernel choices would be more appropriate for the data set that we used. For example, Rasmussen and Williams (Rasmussen 
and Williams, 2006) have used a combination of several base kernels to model (as a function of time) the non-stationary process of the $\mathrm{CO}_{2}$ concentration in the atmosphere on the Mauna Loa dataset. To model the long term rising trend, they used a squared exponential $\left(k_{S E}\right)$ covariance term instead of the linear kernel that we used in this work. Their model provides an excellent fit to the data, but it requires specialized knowledge, trial and error. To cope with these difficulties, Duvenaud et al. (2013) proposed a grammar and an automated search for the kernel structure using a limited number of base kernels. Their method was shown to produce models with excellent fit to the data without the need of the human expert. The drawback is an increased computational cost. We have tested this method, and we have found that the sum of two rational quadratic base kernels produces, in some cases, slightly better results than the kernel used in this work. Moreover, the addition of more base kernels did not improve the model substantially.

\subsection{Software}

In this work we have used the Gaussian Processes for Machine Learning (GPML) toolbox of Rasmussen and Nickisch (2010). The toolbox is freely available under the FreeBSD license and it is fully compatible with both Matlab 7.x (The MathWorks Inc., 2014) and GNU Octave 3.2.x (Octave community, GNU/Octave, 2014). The GPML toolbox includes a library of different covariance and mean functions. In this work, we have used a zero mean function (meanZero) and a combination of kernels using the sum (covSum) of base covariance functions (linear and rational quadratic). The choice of the covariance function is described in Section 4.3. In this work we used Gaussian likelihood (likGauss), thus the inference can be computed exactly using simple algebraic operations (infExact). Moreover, the GPML 
toolbox implements approximate inference algorithms for GP such as Expectation Propagation, the Laplace Approximation and Variational Bayes for a wide class of likelihood functions. We optimized over the hyperparameters, by minimizing the negative log marginal likelihood w.r.t. the hyperparameters using the conjugate gradients (CG) optimiser (minimize.m).

The major limitation of GP regression with exact inference is the computational complexity. In this work the training periods vary from one day to two weeks which corresponds, in the case of half hourly averaged data, to training vector lengths between $2 \times 24=48$ and $2 \times 24 \times 14=672$. The computation with these vector lengths is feasible using a normal computer. On the other hand, in the case of five minutes data, the length of the training vectors varies between $12 \times 24=288$ and $12 \times 24 \times 14=4032$. With these lengths, the dense matrix computations, required by the exact inference, become slow. To cope with this problem we used the FITC approximation (Snelson and Ghahramani, 2006) provided by the GPML toolbox (infFITC). The FITC approximation replaces the exact covariance matrix $\mathbf{K}$ (full GP) with a low-rank plus diagonal matrix $\tilde{\mathbf{K}}=\mathbf{Q}+\operatorname{diag}(\mathbf{K}-\mathbf{Q})$ where $\mathbf{Q}=\mathbf{K}_{\mathbf{u}}{ }^{\top} \mathbf{K}_{\mathbf{u u}}{ }^{-\mathbf{1}} \mathbf{K}_{\mathbf{u}}$. The matrices $\mathbf{K}_{\mathbf{u u}}$ and $\mathbf{K}_{\mathbf{u}}$ contain covariances and cross-covariances of and between inducing inputs $\mathbf{u}^{i}$ and data points $\mathbf{x}^{j}$. The FITC approximation reduces the computational cost to $\mathcal{O}\left(m^{2} n\right)$ in the training phase and to $\mathcal{O}\left(m^{2}\right)$ in the prediction phase $(m \ll n)$. In the supplementary material we show that the full GP models at half hour resolution and the GP models that use the FITC approximation have similar performances. In this case, the inducing points $\mathbf{u}^{i}$ consist of randomly selected $10 \%$ of the total training points - the number of inducing points varies between five (one day of training) and 60 (14 days of training). In the case of five minute resolution models (Section 6.5), because of the higher 
number of training points, we have used $1 \%$ of the total training points as inducing points.

\section{Model evaluation}

In order to evaluate the model we have followed the steps suggested by Bennett et al. (2013). First of all, we have divided the dataset at each site into two disjoint datasets. The data collected during the first two weeks of the measurement campaign have been used as training set $\left(\mathcal{D}_{\text {train }}\right)$. The data collected during the third and fourth week of the measurement campaign have been used as unseen data to evaluate the proposed model $\left(\mathcal{D}_{\text {eval }}\right)$. The training inputs are the concentration measurements of $\mathrm{NO}, \mathrm{NO}_{2}, \mathrm{CO}$ and $\mathrm{O}_{3}$; the training targets are the simultaneous UFP number concentrations. In the prediction phase, the inputs of the model are again measurements of $\mathrm{NO}, \mathrm{NO}_{2}, \mathrm{CO}$ and $\mathrm{O}_{3}$ (of the evaluation period) and the output of the model is the predicted UFP number concentration. The predicted UFP number concentrations are then compared with the measured ones (unseen data). At site 2, the evaluation dataset is limited to 9 days due to monitor malfunctioning. As second step, we have used the highest marginal likelihood (ML), and the lowest deviance information criteria (DIC) - based on the training data only - to select the models that have at the same time a good fit and a low complexity. At this stage, we have compared models (at half hour resolution) based on the maximum length (14 days) of training. In the third step, we have evaluated the selected models in terms of their ability to predict unseen measurements. We have used the $\mathrm{R}^{2}$ and RMSE metrics because their wide usage aids communication of the model performance. Moreover, we have performed a visual analysis of the model 
performance (scatterplots, QQ plots and contingency table), and we have tested the model hypotheses. In a further step, we have extended the quantitative evaluation of the models to different amounts of training data and to higher time resolution (five minutes) models.

\subsection{Metrics}

\subsubsection{Marginal Likelihood (ML)}

The log marginal likelihood Eq. (13) is:

$$
\log p(\mathbf{y} \mid \mathbf{x}, \boldsymbol{\theta})=-\frac{1}{2} \mathbf{y}^{\top} \mathbf{K}_{y}^{-1} \mathbf{y}-\frac{1}{2} \log \left|\mathbf{K}_{y}\right|-\frac{n}{2} \log 2 \pi,
$$

where $\mathbf{K}_{y}=k(\mathbf{X}, \mathbf{X})+\sigma_{n}^{2} \mathbf{I}$ is the covariance matrix for the noisy targets $\mathbf{y}$. The first term $\left(-\frac{1}{2} \mathbf{y}^{\top} \mathbf{K}_{y}^{-1} \mathbf{y}\right)$ gives a measure of the quality of the model fit. It is the only term that involves observed targets. The second term $\left(\log \left|\mathbf{K}_{y}\right|\right)$ is a complexity penalty term, which measures and penalizes the complexity of the model. The third term $\left(\frac{n}{2} \log 2 \pi\right)$ is a $\log$ normalization term. Models with a higher ML should be preferred to models with a lower ML.

In GP regression the trade-off between penalty and data fit is automatic, and therefore automatically incorporates Occam's razor (Rasmussen and Ghahramani, 2001), simplifying the training.

\subsubsection{Deviance Information Criterion (DIC)}

The Deviance Information Criterion (DIC) (Spiegelhalter et al., 2002) is a Bayesian generalization of the Aikake information criterion for posterior predictive model comparison. Like many other criteria it is a compromise between data fit and model complexity. Models with a lower DIC should be preferred to models with a higher DIC:

$$
\mathrm{DIC}=\overline{D(\boldsymbol{\theta})}+p_{D}=D(\overline{\boldsymbol{\theta}})+2 p_{D}
$$


where $\overline{D(\boldsymbol{\theta})}$ is the posterior mean of the deviance $(D(\boldsymbol{\theta})), D(\overline{\boldsymbol{\theta}})$ is the deviance evaluated at the posterior mean of the parameters and $p_{D}$ is the effective number of parameters, which penalizes the complexity of the model:

$$
\begin{gathered}
\overline{D(\boldsymbol{\theta})}=\mathbb{E}_{\boldsymbol{\theta} \mid \mathbf{y}}[-2 \log (p(\mathbf{y} \mid \boldsymbol{\theta}))]+2 \log (f(\mathbf{y})), \\
D(\boldsymbol{\theta})=2(\log (f(\mathbf{y}))-\log (p(\mathbf{y} \mid \boldsymbol{\theta}))), \\
p_{D}=\overline{D(\boldsymbol{\theta})}-D(\overline{\boldsymbol{\theta}}),
\end{gathered}
$$

where $p(\mathbf{y} \mid \boldsymbol{\theta})$ is the likelihood function, and $f(\mathbf{y})$ is a standardizing term that is a function of the data alone.

\subsubsection{Coefficient of determination $\left(R^{2}\right)$}

The coefficient of determination $\mathrm{R}^{2}$ indicates the fraction of variance of observations explained by the model:

$$
\mathrm{R}^{2}=1-\frac{\sum_{m}^{M}\left(y_{m}-y_{*}\right)^{2}}{\sum_{m}^{M}\left(y_{m}-\bar{y}\right)^{2}}
$$

where $y_{m}$ and $y_{*}$ are the measured and predicted UFP; $\bar{y}$ is the mean of the observed UFP; $M$ is the number of the evaluation measurements.

\subsubsection{Root Mean Square Error (RMSE)}

The root mean square error (RMSE) is calculated as the difference between the measured UFP number concentrations and the predicted ones:

$$
\mathrm{RMSE}=\sqrt{\frac{1}{M} \sum_{m=1}^{M}\left(y_{m}-y_{*}\right)^{2}}
$$

where $y_{m}$ and $y_{*}$ are the measured and predicted UFP number concentrations and $M$ is the number of the evaluation measurements. 


\section{Results and discussion}

In this section, we first show the model selection results, in terms of the ML and DIC (Section 6.1). Then, we evaluate and discuss the performance of the GP models that use $\mathrm{NO}$ and $\mathrm{NO}_{2}$ as covariates (model selected) by comparing the estimated UFP number concentrations with the measured ones (Section 6.2). Then we discuss the adequacy of the model hypotheses (Section 6.3). The results in Sections 6.1-6.3 are based on a half hour resolution and 14 days of training. We conclude by evaluating the model on different amounts of training data at half hour resolution (Section 6.4) and its performance at five minutes resolution (Section 6.5). All the results are based on log-transformed and standardised data with zero mean and unit variance.

\subsection{Model selection}

We have used the highest ML, and the lowest DIC - based on the training data only - to select the models that have at the same time the best fit and the lowest complexity. Both metrics (Table 2) reveal that the GP models that use $\mathrm{NO}$ and $\mathrm{NO}_{2}$ as covariates $\left(\mathrm{GP}\left(\mathrm{NO}, \mathrm{NO}_{2}\right)\right.$, in bold) outperform the GP models that use $\mathrm{O}_{3}$ or $\mathrm{CO}$ as covariates $\left(\mathrm{GP}\left(\mathrm{O}_{3}\right)\right.$ and $\mathrm{GP}(\mathrm{CO})$, respectively) and the Bayesian linear models that use $\mathrm{NO}$ and $\mathrm{NO}_{2}$ as covariates $\left(\operatorname{Lin}\left(\mathrm{NO}, \mathrm{NO}_{2}\right)\right)$.

Considering the high cost of the pollutant monitors (in the order of ten to fifteen thousands Euro), we have evaluated models that use covariates from only one monitor: $\mathrm{NO}$ and $\mathrm{NO}_{2}$ are measured by the same monitor, and the inclusion of the $\mathrm{O}_{3}$ and $\mathrm{CO}$ covariates requires the inclusion of one monitor for each covariate, increasing the costs of the instrumentation and maintenance. 
Table 2: Evaluation of the models at half hour resolution and 14 days of training in terms of ML, DIC, $\mathrm{R}^{2}$ RMSE. In the DIC column, the numbers in brackets are the effective number of parameters.

\begin{tabular}{|c|c|c|c|c|c|}
\hline & Model & $\mathrm{ML}$ & DIC & $\mathrm{R}^{2}$ & RMSE \\
\hline \multirow{4}{*}{ Site 1} & $\mathrm{GP}(\mathrm{CO})$ & -601 & 1048(49.59) & 0.574 & 0.654 \\
\hline & $\mathrm{GP}\left(\mathrm{O}_{3}\right)$ & -465 & $882(22.09)$ & 0.575 & 0.672 \\
\hline & $\mathbf{G P}\left(\mathbf{N O}, \mathrm{NO}_{2}\right)$ & -131 & $210(26.41)$ & 0.897 & 0.342 \\
\hline & $\operatorname{Lin}\left(\mathrm{NO}, \mathrm{NO}_{2}\right)$ & -313 & $405(1.96)$ & 0.876 & 0.427 \\
\hline \multirow{4}{*}{ Site 2} & $\mathrm{GP}(\mathrm{CO})$ & - & - & - & - \\
\hline & $\mathrm{GP}\left(\mathrm{O}_{3}\right)$ & -436 & $897(46.16)$ & 0.584 & 0.671 \\
\hline & $\mathrm{GP}\left(\mathrm{NO}, \mathrm{NO}_{2}\right)$ & -214 & $339(50.06)$ & 0.856 & 0.434 \\
\hline & $\operatorname{Lin}\left(\mathrm{NO}, \mathrm{NO}_{2}\right)$ & -260 & $460(1.97)$ & 0.828 & 0.466 \\
\hline \multirow{4}{*}{ Site 3} & $\mathrm{GP}(\mathrm{CO})$ & -734 & $1478(6.83)$ & 0.359 & 0.811 \\
\hline & $\mathrm{GP}\left(\mathrm{O}_{3}\right)$ & -569 & $1081(10.21)$ & 0.671 & 0.583 \\
\hline & $\mathrm{GP}\left(\mathrm{NO}, \mathrm{NO}_{2}\right)$ & -414 & $767(36.96)$ & 0.867 & 0.374 \\
\hline & $\operatorname{Lin}\left(\mathrm{NO}, \mathrm{NO}_{2}\right)$ & -450 & $855(2.01)$ & 0.839 & 0.411 \\
\hline
\end{tabular}

\subsection{Model evaluation}

In this section, we evaluate the selected $\mathrm{GP}\left(\mathrm{NO}, \mathrm{NO}_{2}\right)$ models (Section 6.1), in terms of the model's ability to predict unseen measurements.

First of all, according to the $\mathrm{R}^{2}$ and RMSE metrics (Table 2), the results of the GP and linear models that use $\mathrm{NO}$ and $\mathrm{NO}_{2}$ show a good correspondence between the modelled and the measured values. Moreover, they confirm the results of the ML and DIC metrics (Table 2), showing that the GP models that use $\mathrm{NO}$ and $\mathrm{NO}_{2}$ as covariates (in bold) outperform the others.

Figure 5 shows the time series, scatterplots and QQ plots of the modelled 
and the measured UFP number concentrations for the three different sites. At all three sites, the models explain between $86 \%$ (site 2) and $90 \%$ (site 1) of the variance. From the scatterplots together with the QQ plots (second and third column in Figure 5, respectively), we can observe that, at all three sites, the models tend to overestimate high values of the UFP number concentrations. Moreover, the model tends to underestimate low values at site 2 , and underestimates between the first and second quantiles at site 3 .

It should be emphasized that these deviations are not substantial, and the estimated distributions seem to describe the measurements well. In particular, the models do not tend to underestimate the risk of high concentrations.

In air pollution applications, it is useful to report the model's behaviour in the presence of high pollution episodes (when the pollution levels exceed a specified threshold). As described in Section 1, UFP is not a regulated pollutant, and therefore, there are no concentration limits set by the law. In Table 3, we have used three different thresholds: (i) one coincides with the first quartile of the measured UFP (Alarm=Q1); (ii) one coincides with the median of the measured UFP (Alarm=Q2); (iii) one coincides with the third quartile of the measured UFP (Alarm=Q3). The contingency table (Table 3) reports the occurrences and rates in which: (i) measured and modelled UFP were both above the threshold (true positives, tp); (ii) measured and modelled UFP were both below the threshold (true negatives, tn); (iii) the occurrences and rates of alarms missed by the model (false negatives, fn); (iv) the occurrences and rates of false alarms (false positives, fp). The critical value for the human health is the percentage of missed alarms (fn, in bold). A perfect model would have data only on the main diagonal. The probability of correct detection $-(\mathrm{tp}+\mathrm{tn}) /(\mathrm{tp}+\mathrm{tn}+\mathrm{fn}+\mathrm{fp})-$, which varies between 0.87 

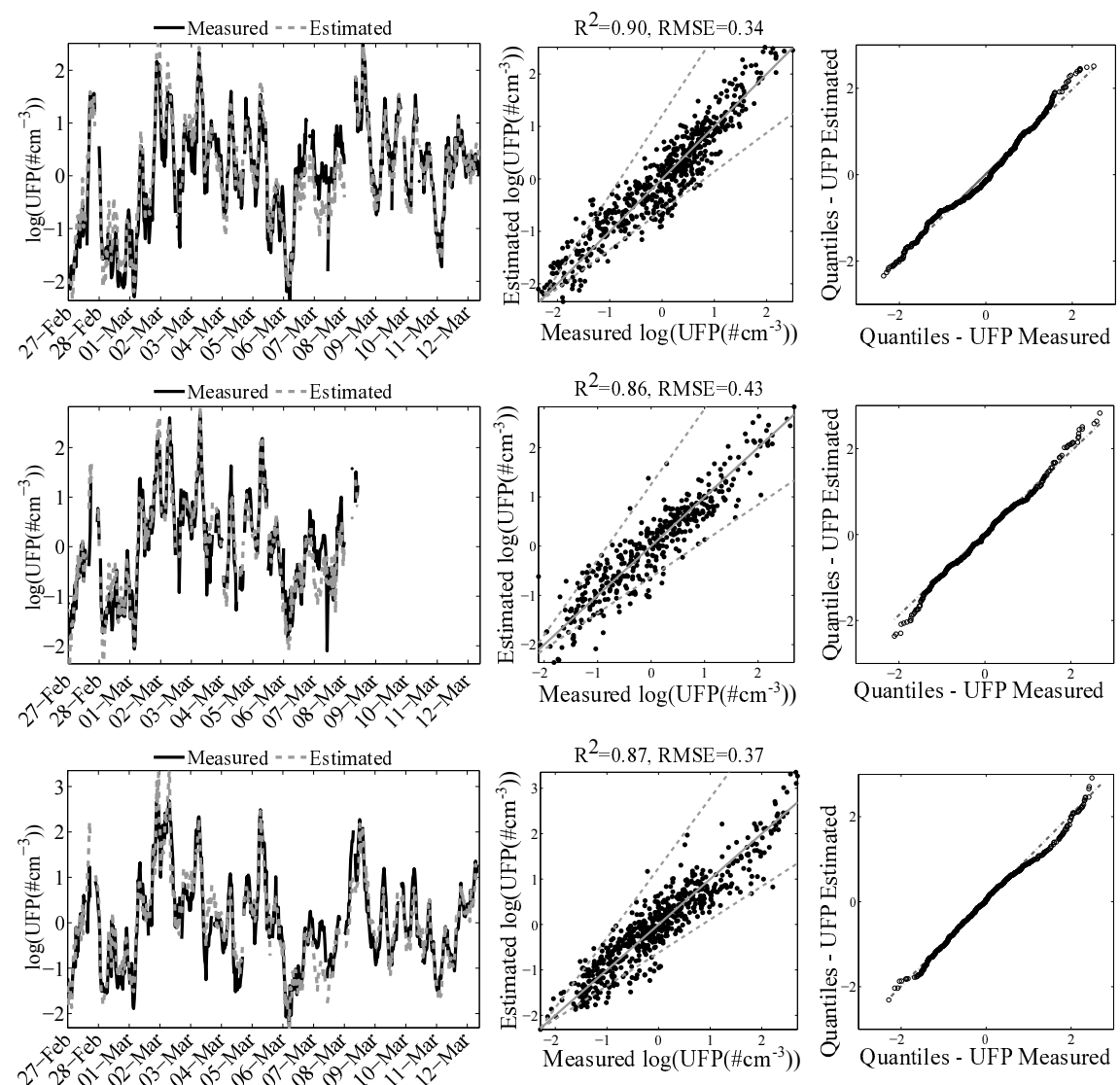

Figure 5: The left column shows the time series plots of the predicted and the measured UFP number concentrations. The dashed gray line is the predicted UFP number concentration and the black line is the measured UFP number concentration relative to the evaluation period $\left(\mathcal{D}_{\text {eval }}\right)$. The middle column shows the scatterplots, $\mathrm{R}^{2}$ and RMSE between the predicted and measured UFP number concentrations. The gray lines have slope 1 and an intercept of 0 (ideal case, when the predicted and measured values are equal). The dashed gray lines delimit the FAC2 area. The right column shows the QQ plots between the predicted and measured UFP number concentrations. The top row refers to site 1 , the middle row refers to site 2 and the bottom row refers to site 3 . 
(site 2 and Alarm=Q1) and 0.92 (site 1, Alarm=Q1 and Alarm=Q3), shows a good overall accuracy of the models.

Table 3: Contingency table for the GP models, $\mathrm{GP}\left(\mathrm{NO}, \mathrm{NO}_{2}\right)$ at half hour resolution and 14 days of training. The numbers next to $t p, t n, f n$ and $\mathrm{fp}$ are the occurrences and in brackets their percentage.

\begin{tabular}{|c|c|c|c|c|c|c|}
\hline & \multicolumn{2}{|c|}{ Alarm=Q1 } & \multicolumn{2}{|c|}{ Alarm=Q2 } & \multicolumn{2}{|c|}{ Alarm=Q3 } \\
\hline & $\begin{array}{l}\mathrm{tp}=423 \\
(95 \%)\end{array}$ & $\begin{array}{l}\mathrm{fp}=27 \\
(18 \%)\end{array}$ & $\begin{array}{l}\mathrm{tp}=254 \\
(85 \%)\end{array}$ & $\begin{array}{l}\mathrm{fp}=24 \\
(8 \%)\end{array}$ & $\begin{array}{l}\mathrm{tp}=125 \\
(85 \%)\end{array}$ & $\begin{array}{l}\mathrm{fp}=23 \\
(5 \%)\end{array}$ \\
\hline \multirow[t]{2}{*}{ Site 1} & $\begin{array}{l}\mathrm{fn}=23 \\
(5 \%)\end{array}$ & $\begin{array}{l}\operatorname{tn}=121 \\
(82 \%)\end{array}$ & $\begin{array}{l}\mathrm{fn}=44 \\
(15 \%)\end{array}$ & $\begin{array}{l}\operatorname{tn}=272 \\
(92 \%)\end{array}$ & $\begin{array}{l}f n=23 \\
(15 \%)\end{array}$ & $\begin{array}{l}\operatorname{tn}=423 \\
(95 \%)\end{array}$ \\
\hline & $\begin{array}{l}\mathrm{tp}=281 \\
(93 \%)\end{array}$ & $\begin{array}{l}\mathrm{fp}=30 \\
(30 \%)\end{array}$ & $\begin{array}{l}\mathrm{tp}=179 \\
(89 \%)\end{array}$ & $\begin{array}{l}\mathrm{fp}=20 \\
(10 \%)\end{array}$ & $\begin{array}{l}\mathrm{tp}=80 \\
(79 \%)\end{array}$ & $\begin{array}{l}\mathrm{fp}=20 \\
(7 \%)\end{array}$ \\
\hline \multirow[t]{2}{*}{ Site 2} & $\begin{array}{l}\mathrm{fn}=21 \\
(7 \%)\end{array}$ & $\begin{array}{l}\operatorname{tn}=70 \\
(70 \%)\end{array}$ & $\begin{array}{l}f n=22 \\
(11 \%)\end{array}$ & $\begin{array}{l}\operatorname{tn}=181 \\
(90 \%)\end{array}$ & $\begin{array}{l}f n=21 \\
(21 \%)\end{array}$ & $\begin{array}{l}\operatorname{tn}=281 \\
(93 \%)\end{array}$ \\
\hline & $\begin{array}{l}\mathrm{tp}=429 \\
(94 \%)\end{array}$ & $\begin{array}{l}\mathrm{fp}=29 \\
(19 \%)\end{array}$ & $\begin{array}{l}\mathrm{tp}=273 \\
(90 \%)\end{array}$ & $\begin{array}{l}\mathrm{fp}=41 \\
(14 \%)\end{array}$ & $\begin{array}{l}\mathrm{tp}=123 \\
(81 \%)\end{array}$ & $\begin{array}{l}\mathrm{fp}=32 \\
(7 \%)\end{array}$ \\
\hline Site 3 & $\begin{array}{l}f n=27 \\
(6 \%)\end{array}$ & $\begin{array}{l}\mathrm{tn}=122 \\
(81 \%)\end{array}$ & $\begin{array}{l}\mathrm{fn}=31 \\
(10 \%)\end{array}$ & $\begin{array}{l}\operatorname{tn}=262 \\
(86 \%)\end{array}$ & $\begin{array}{l}f n=29 \\
(19 \%)\end{array}$ & $\begin{array}{l}\operatorname{tn}=423 \\
(93 \%)\end{array}$ \\
\hline
\end{tabular}

Dawid (1984) proposed the use of the probability integral transform (PIT) for an adequate probabilistic description of the estimated distributions. The PIT is the value that the predictive cumulative distribution function attains at the value that materializes. If the predictive distribution is continuous, and the observation is drawn from the predictive distribution (desirable case), the PIT follows a standard uniform distribution. The histograms of the PIT values (Figure 6) confirm the uniformity of the PIT at all three sites, as deviations from uniformity would point to failures and model deficiencies: (i) U-shaped histograms indicate under-dispersed pre- 

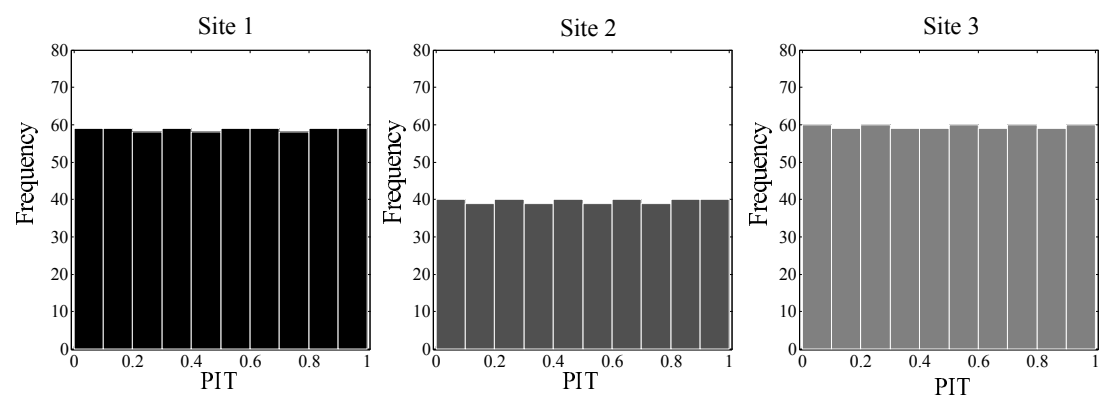

Figure 6: PIT histograms of the $\mathrm{GP}\left(\mathrm{NO}, \mathrm{NO}_{2}\right)$ models at half hour resolution and 14 days of training. From left to right they refer to sites 1, 2 and 3, respectively.

dictive distributions; (ii) hump or inverse-U shaped histograms point at over-dispersion; and (iii) skewed histograms indicate biased tendencies.

\subsection{Test of the model hypotheses}

In the formulation of the model, we have assumed that the errors (Eq. (3)) are (i) independent, (ii) normally distributed with zero mean and constant standard deviation. The left-hand side of Figure 7 shows that the autocorrelations of the residuals remain close to zero for almost all non-zero lags, and therefore, the assumption of independent residuals is reasonable. In the right-hand side of Figure 7, we have plotted the QQ plots of the residuals against the theoretical normal distribution to check whether or not it is reasonable to assume that the random errors have been drawn from a normal distribution. The plots show that the points lie close to the straight line (at site 2 there are long tails at both ends of the data distribution), and therefore, it is reasonable to assume that the random errors are drawn from approximately normal distributions. Moreover, the Lilliefors test at the $95 \%$ confidence level confirmed these results. 
Site 1
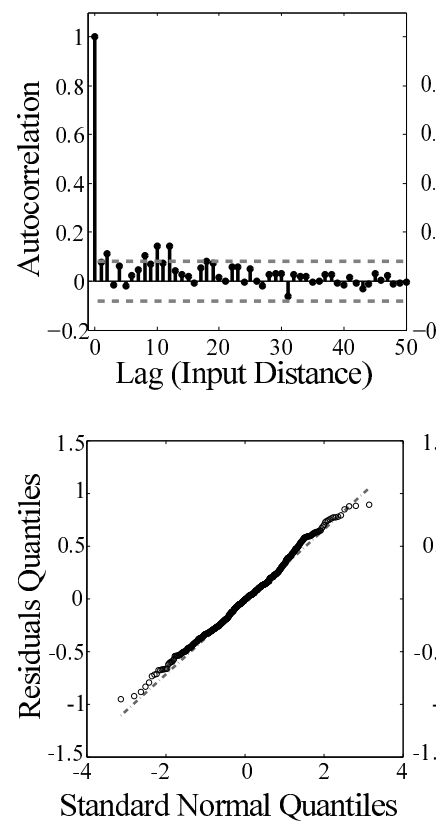

Site 2
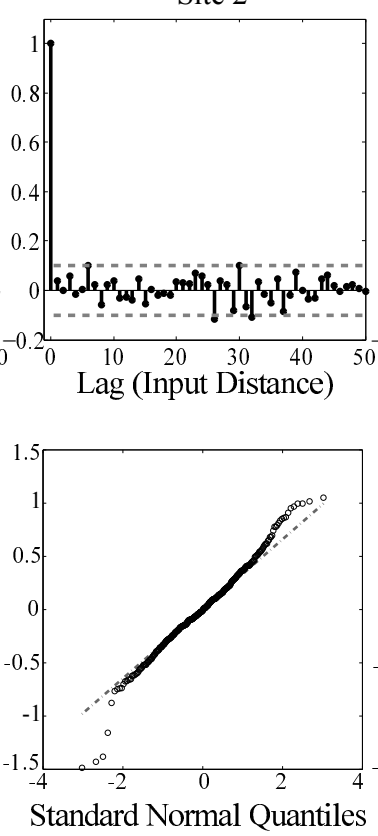

Site 3
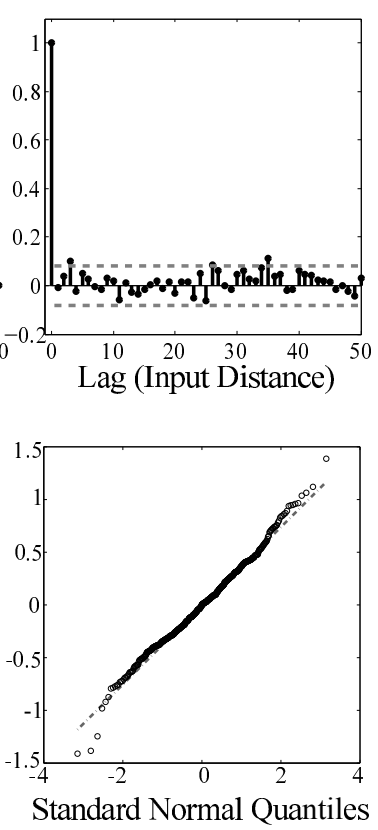

Figure 7: Residual analysis of the $\mathrm{GP}\left(\mathrm{NO}, \mathrm{NO}_{2}\right)$ models at half hour resolution and 14 days of training. Top row shows the autocorrelation of the residuals and bottom row shows the quantile-quantile plots of the residuals against the theoretical normal distribution. 


\subsection{Training length}

In practical situations such as designing the measurement campaign and planning the facilities needed, it is useful to know how the prediction model performs according to the amount of data used for training. In Figure 8, the model performances, for each site, are evaluated on different days of training. One day of training refers to the day before the first day of evaluation, two days of training means two days before the first day of evaluation and so on up to 14 days. The evaluation data set $\left(\mathcal{D}_{\text {eval }}\right)$ is the one described in Section 5, and it comprises two weeks of measurements.

The results show a good correspondence of the models $\mathrm{GP}\left(\mathrm{NO}, \mathrm{NO}_{2}\right)$ and Linear $\left(\mathrm{NO}, \mathrm{NO}_{2}\right)$ with the measured values of UFP for all amounts of training data. This is probably due to the strong correlation of UFP with $\mathrm{NO}$ and $\mathrm{NO}_{2}$, which in its turn can be related to the high share of diesel vehicles in the Belgian car fleet.

More in detail, the ML and DIC plots show that the GP models outperform the Bayesian linear models for all amounts of training data. According to the $\mathrm{R}^{2}$ metric, the GP models at sites 1 and 2 outperform the linear models for training periods longer than four days and at site 3 for training periods longer than six days. The RMSE values at sites 2 and 3 agree with the $\mathrm{R}^{2}$ results, and at site 1 the RMSE values of the GP model are lower than the ones of the linear model for all the training lengths.

In the case of the GP models, the $\mathrm{R}^{2}$ and RMSE plots show that the model performance increases with the training length. It seems that a training period of at least seven days (in which at least two days correspond to weekend days) is suitable (in terms of a trade-off between costs and model performance) to let the model learn the UFP number concentration dynamics in different typologies of traffic. On the other hand, the ML and DIC 


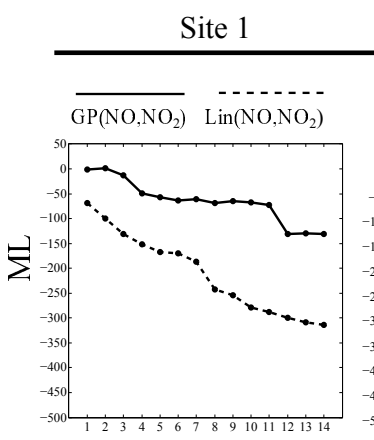

Site 2

Site 3
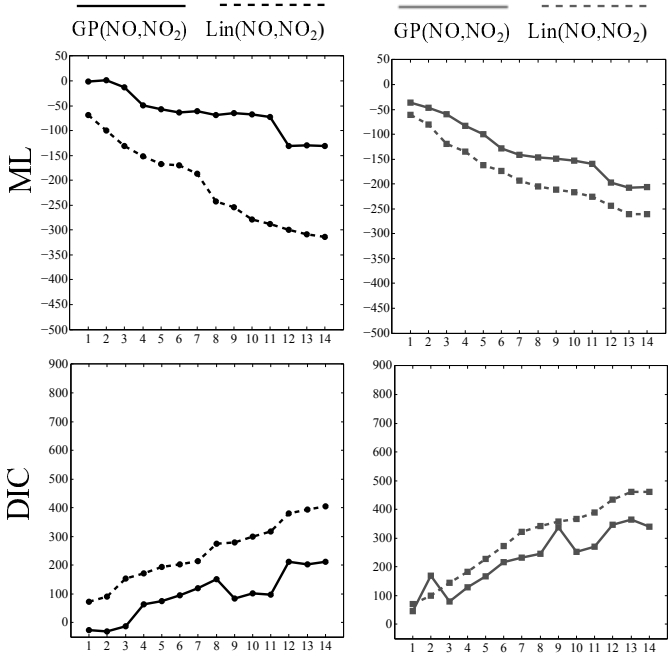

$\overline{\mathrm{GP}\left(\mathrm{NO}, \mathrm{NO}_{2}\right)} \quad \mathrm{Lin}\left(\mathrm{NO}, \mathrm{NO}_{2}\right)$
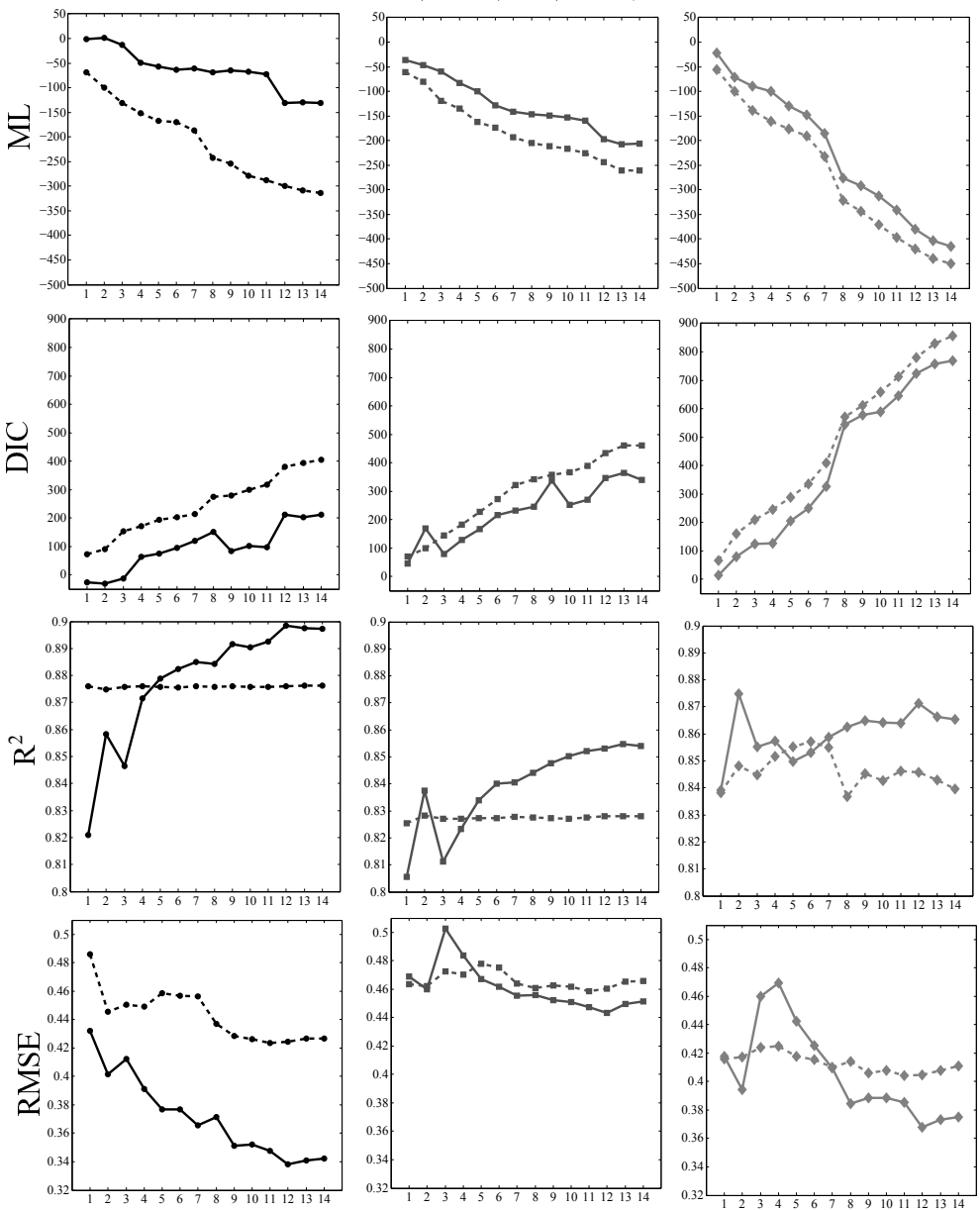

Day of Training

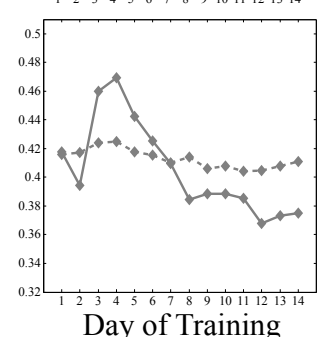

Figure 8: Performances of the $\mathrm{GP}\left(\mathrm{NO}, \mathrm{NO}_{2}\right)$ and $\mathrm{Lin}\left(\mathrm{NO}, \mathrm{NO}_{2}\right)$ models at half hour resolution evaluated on different days of training. First row: marginal likelihood (ML); second row: deviance information criterion (DIC); third row: coefficient of determination $\left(R^{2}\right)$; fourth row: root mean square error (RMSE). One day of training refers to the day before the first day of evaluation, two days of training means two days before the first day of evaluation and so on up to 14 days. 
values get worse when increasing the amount of training data, suggesting that the models that use only one day of training seem to combine a good model fit with a low model complexity (higher ML and lower DIC).

The ML and DIC results (computed on the training data sets) agree with the $\mathrm{R}^{2}$ and RMSE results (computed on the evaluation data) of the Bayesian linear model: the $\mathrm{R}^{2}$ and RMSE values are similar for all the training lengths. On the other hand, they do not agree with the $\mathrm{R}^{2}$ and RMSE results of the GP models: the two metrics show improvements in the model performance with longer training periods. Moreover, in the case of models computed using one day of training, the ML and DIC metrics indicate that the GP models outperform the linear model; on the other hand, the evaluations on the independent dataset $\left(\mathrm{R}^{2}\right.$ and RMSE) reveal that the GP models have a worse model performance than the linear models.

We have also noticed that both the ML and DIC strongly depend on the data normalization used. In this paper, we have log-transformed and standardized the data with zero mean and unit standard deviation. With this data normalization, Figure 8 reveals that the ML and DIC metrics get worse when increasing the amount of training data. On the other hand, for logtransformed data (without normalization) and log-transformed data scaled between zero and one - Figures A.11 and A.12 in the supplementary material section - we show that the ML and DIC metrics have opposite trends: they improve when increasing the amount of training data. Therefore, we are not confident to use the ML and DIC to point out how much training data is "enough" to get the best increase in model fit, but we suggest using the ML and DIC metrics to compare models that use the same amount of data. 


\subsection{Models at five minute resolution}

All the above results are based on half hour resolution. Considering the high variability of the UFP number concentrations, it is also interesting to have models with a higher time resolution. Figure 9 shows the results obtained for models that give a prediction every five minutes using different training lengths.

The results of models at five minutes resolution, as in the half hour models, show a good correspondence of the UFP values for both $\operatorname{GP}\left(\mathrm{NO}, \mathrm{NO}_{2}\right)$ and $\operatorname{Lin}\left(\mathrm{NO}, \mathrm{NO}_{2}\right)$ models with the measured values. At sites 1 and 3 , the GP models explain up to $86 \%$ of the variance and at site 2 the GP model explains $78 \%$ of the variance. Similarly to the half hour resolution, the ML plots show that the GP models outperform the Bayesian linear model for all amounts of training data. According to the $\mathrm{R}^{2}$ metric, the GP models at sites 1 and 2 outperform the linear models for training periods longer than four days and at site 3 for training periods longer than seven days. Moreover, the RMSE values have slightly worse values (due to the increased dynamics of the particles) and similar trends to the ones at half hour resolution.

\section{Conclusions}

In this paper we have evaluated the potential to predict UFP number concentrations from simultaneously measured $\mathrm{NO}, \mathrm{NO}_{2}, \mathrm{CO}$ and $\mathrm{O}_{3}$ concentrations by treating this prediction problem as a supervised regression problem using Gaussian process (GP) regression. The proposed model uses $\mathrm{NO}, \mathrm{NO}_{2}, \mathrm{CO}$ and $\mathrm{O}_{3}$ concentration measurements as covariates. The model is based on non-parametric Bayesian inference techniques and it does not require any a priori assumption about the functional form of the relationship 


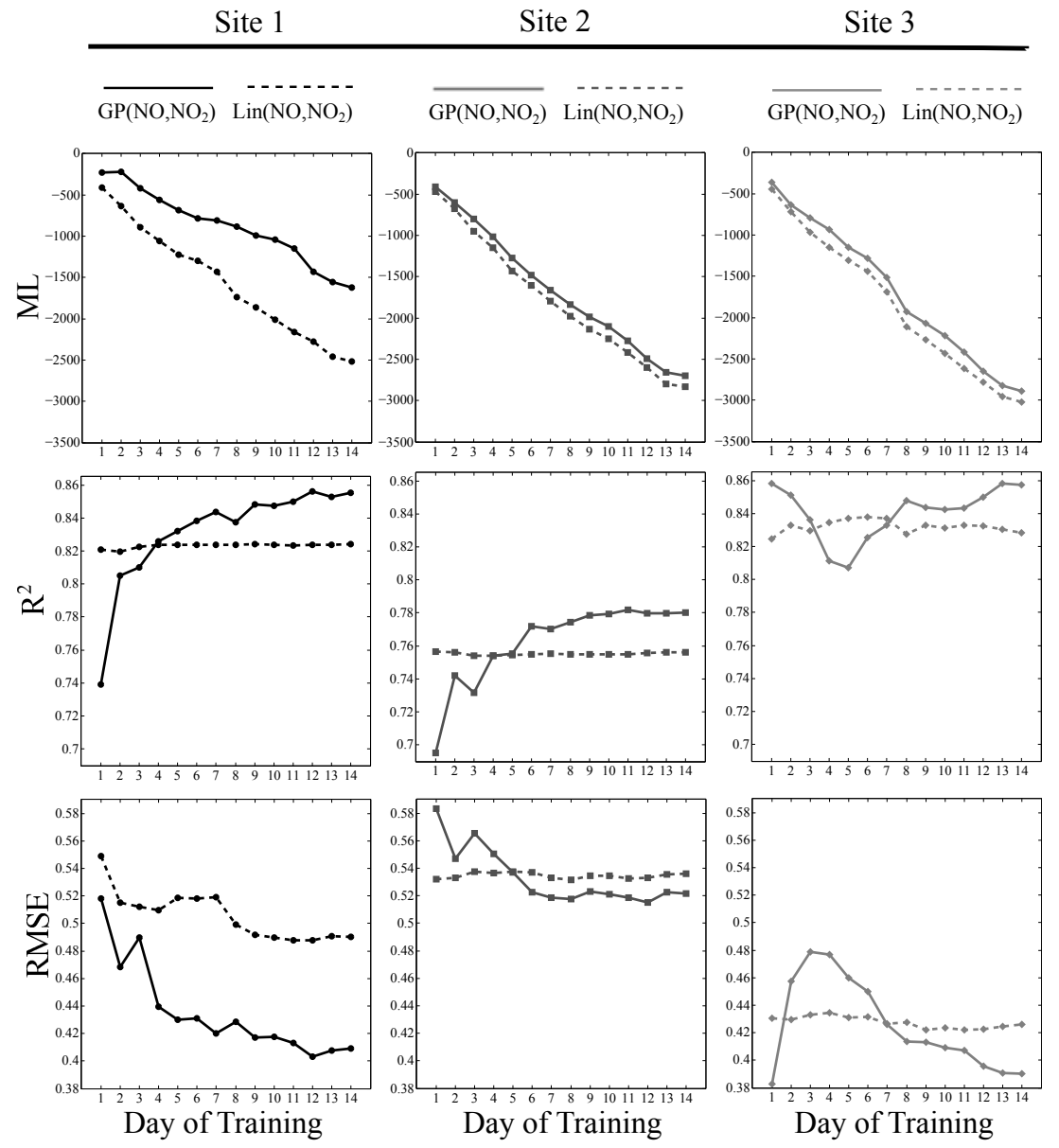

Figure 9: Performances of the $\mathrm{GP}\left(\mathrm{NO}, \mathrm{NO}_{2}\right)$ and $\mathrm{Lin}\left(\mathrm{NO}, \mathrm{NO}_{2}\right)$ models at five minutes resolution evaluated on different days of training. First row: ML; second row: $\mathrm{R}^{2}$; third row: RMSE. One day of training refers to the day before the first day of evaluation, two days of training means two days before the first day of evaluation and so on up to 14 days. 
between the input and target variables.

We have evaluated the model using the data recorded during a one-month measurement campaign (in winter) in which UFP number concentrations and relevant pollutants $\left(\mathrm{NO}, \mathrm{NO}_{2}, \mathrm{CO}\right.$ and $\left.\mathrm{O}_{3}\right)$ were gathered simultaneously at three sampling locations close to the traffic in an urban area in Antwerp, Belgium. The dataset has been divided into two disjoint parts: one to learn the hyper-parameters of the model and the other one to evaluate the prediction performance.

We have found that the GP models and the Bayesian linear models that use $\mathrm{NO}$ and $\mathrm{NO}_{2}$ as covariates outperform the models that use $\mathrm{CO}$ and $\mathrm{O}_{3}$ as covariates. This is probably due to the strong correlation of UFP with $\mathrm{NO}$ and $\mathrm{NO}_{2}$, which in turn can be related to the high share of diesel vehicles in the Belgian car fleet. The GP models outperform the Bayesian linear models at all sites. The results show a good correspondence between the modelled and the measured values, and the models are able to explain up to $90 \%$ of the variance. Moreover, the deviations between the modelled and measured values are not substantial, and the models do not tend to underestimate the risk of high concentrations.

We have also evaluated the models on different amounts of training data and we have found that a training period of at least seven days (in which at least two days correspond to weekend days) is a suitable period (in terms of a trade-off between costs and model performance) to let the models learn the UFP number concentration dynamics in different typologies of traffic, giving thus a practical guideline to plan measurement campaigns. The practical implication of this work lies in the fact that NOx monitors that are available in existing monitoring networks can be used for prediction of simultaneous UFP number concentrations using a limited set of local training data. 
The approach has its limitations as well. For instance, there is no guarantee that the proposed model structure is optimal, but different covariates (e.g. traffic and meteorological data) can easily be added to it because of its structure. The proposed method was successfully tested simultaneously at three different locations, which indicates that it can be used at other locations as well. However, site specific data is needed for model evaluation at other sites.

Furthermore, the lower cut-off limit used for the modelling purposes here $(25 \mathrm{~nm})$ does not account for the nucleation mode particles, which are volatile and much more dynamic. It would be interesting to evaluate the model on a data set that includes particles smaller than $25 \mathrm{~nm}$. A further limitation of the data set used is that it is one month long, and considering that half has been used for training, the evaluation has been done on the remaining period. We cannot assess for how long the proposed model will perform satisfactorily and how often retraining will have to be performed. It could be interesting, since pollutants can behave differently, to extend this work to study the model performances across different seasons.

Future modelling attempts could include the investigation of the use of locally adaptive kernels using a mixture of different models, each one used in a local region of the input space. This kind of model, known as a mixture of experts (Jacobs et al., 1991), has the advantage of reducing the computational complexity. More importantly, the model has the capability to adapt itself locally to the multimodal characteristics of the pollutants time series, thus being able to adapt to sudden (abrupt) changes of the aerosol concentrations. A further interesting study could be to predict UFP number concentrations at different locations using training data from another location under varying meteorological and traffic conditions. 


\section{Acknowledgment}

This research is part of the IDEA (Intelligent, Distributed Environmental Assessment) project, a 4 year strategic basic research project, financially supported by the IWT-Vlaanderen (Flemish Agency for Innovation by Science and Technology). The authors thank Carl Rasmussen and Hannes Nickisch for making the GPML Toolbox available.

\section{References}

Alvarez, R., Weilenmann, M., Favez, J.Y., 2008. Evidence of increased mass fraction of $\mathrm{NO}_{2}$ within real-world $\mathrm{NOx}$ emissions of modern light vehicles derived from a reliable online measuring method. Atmospheric Environment 42, 4699-4707.

Atkinson, R.W., Fuller, G.W., Anderson, H.R., Harrison, R.M., Armstrong, B., 2010. Urban ambient particle metrics and health: A time-series analysis. Epidemiology 21, 501-511.

Beckx, C., Denys, T., Michiels, H., 2013. Analysis of the Belgian Car Fleet 2012 - Report for the Flemish, the Walloon and the Brussels Capital Region. Technical Report. VITO.

Beddows, D.C., Harrison, R.M., 2008. Comparison of average particle number emission factors for heavy and light duty vehicles derived from rolling chassis dynamometer and field studies. Atmospheric Environment 42, 7954-7966.

Bennett, N.D., Croke, B.F., Guariso, G., Guillaume, J.H., Hamilton, S.H., Jakeman, A.J., Marsili-Libelli, S., Newham, L.T., Norton, J.P., Perrin, 
C., Pierce, S.A., Robson, B., Seppelt, R., Voinov, A.A., Fath, B.D., Andreassian, V., 2013. Characterising performance of environmental models. Environmental Modelling \& Software 40, 1-20.

Bos, I., Jacobs, L., Nawrot, T., de Geus, B., Torfs, R., Int Panis, L., Degraeuwe, B., Meeusen, R., 2011. No exercise-induced increase in serum BDNF after cycling near a major traffic road. Neuroscience Letters 500, $129-132$.

Buonanno, G., Lall, A., Stabile, L., 2009. Temporal size distribution and concentration of particles near a major highway. Atmospheric Environment 43, 1100-1105.

Charron, A., Harrison, R.M., 2003. Primary particle formation from vehicle emissions during exhaust dilution in the roadside atmosphere. Atmospheric Environment 37, 4109-4119.

Clifford, S., Choy, S.L., Hussein, T., Mengersen, K., Morawska, L., 2011. Using the generalised additive model to model the particle number count of ultrafine particles. Atmospheric Environment 45, 5934-5945.

Dawid, A.P., 1984. Present Position and Potential Developments: Some Personal Views: Statistical Theory: The Prequential Approach. Journal of the Royal Statistical Society. Series A (General) 147, 278-292.

Duvenaud, D., Lloyd, J.R., Grosse, R., Tenenbaum, J.B., Ghahramani, Z., 2013. Structure discovery in nonparametric regression through compositional kernel search, in: Proceedings of the 30th International Conference on Machine Learning, pp. 1166-1174. 
Fujitani, Y., Kumar, P., Tamura, K., Fushimi, A., Hasegawa, S., Takahashi, K., Tanabe, K., Kobayashi, S., Hirano, S., 2012. Seasonal differences of the atmospheric particle size distribution in a metropolitan area in Japan. Science of the Total Environment 437, 339-347.

Hagler, G.S.W., Thoma, E.D., Baldauf, R.W., 2010. High-resolution mobile monitoring of carbon monoxide and ultrafine particle concentrations in a near-road environment. Journal of the Air and Waste Management Association 60, 328-336.

de Hartog, J.J., Hoek, G., Peters, A., Timonen, K.L., Ibald-Mulli, A., Brunekreef, B., Heinrich, J., Tiittanen, P., van Wijnen, J.H., Kreyling, W., Kulmala, M., Pekkanen, J., 2003. Effects of fine and ultrafine particles on cardiorespiratory symptoms in elderly subjects with coronary heart disease. American Journal of Epidemiology 157, 613-623.

Heal, M.R., Kumar, P., Harrison, R.M., 2012. Particles, air quality, policy and health. Chem. Soc. Rev. 41, 6606-6630.

Higdon, D., 1998. A process-convolution approach to modelling temperatures in the North Atlantic Ocean. Environmental and Ecological Statistics 5, 173-190.

Holmes, N., Morawska, L., 2006. A review of dispersion modelling and its application to the dispersion of particles: An overview of different dispersion models available. Atmospheric Environment 40, 5902-5928.

Hong, Y.C., Lee, J.T., Kim, H., Ha, E.H., Schwartz, J., Christiani, D.C., 2002. Effects of air pollutants on acute stroke mortality. Environmental Health Perspectives 110, 187-191. 
Hussein, T., Karppinen, A., Kukkonen, J., Hrknen, J., Aalto, P.P., Hmeri, K., Kerminen, V.M., Kulmala, M., 2006. Meteorological dependence of size-fractionated number concentrations of urban aerosol particles. Atmospheric Environment 40, 1427-1440.

Jacobs, L., Nawrot, T., de Geus, B., Meeusen, R., Degraeuwe, B., Bernard, A., Sughis, M., Nemery, B., Int Panis, L., 2010. Subclinical responses in healthy cyclists briefly exposed to traffic-related air pollution: an intervention study. Environmental Health 9, 64-71.

Jacobs, R.A., Jordan, M.I., Nowlan, S.J., Hinton, G.E., 1991. Adaptive Mixtures of Local Experts. Neural Computation 3, 79-87.

Janhäll, S., M. Jonsson, A.s., Molnár, P., A. Svensson, E., Hallquist, M., 2004. Size resolved traffic emission factors of submicrometer particles. Atmospheric Environment 38, 4331-4340.

Keuken, M., Roemer, M., van den Elshout, S., 2009. Trend analysis of urban $\mathrm{NO}_{2}$ concentrations and the importance of direct $\mathrm{NO}_{2}$ emissions versus ozone/NOx equilibrium. Atmospheric Environment 43, 4780-4783.

Kittelson, D., Watts, W., Johnson, J., 2004. Nanoparticle emissions on Minnesota highways. Atmospheric Environment 38, 9-19.

Kittelson, D.B., 1998. Engines and nanoparticles: a review. Journal of Aerosol Science 29, 575-588.

Kulmala, M., Vehkamki, H., Petj, T., Maso, M.D., Lauri, A., Kerminen, V.M., Birmili, W., McMurry, P., 2004. Formation and growth rates of ultrafine atmospheric particles: a review of observations. Journal of Aerosol Science 35, 143-176. 
Kumar, P., Fennell, P., Britter, R., 2008. Measurements of particles in the 5-1000 nm range close to road level in an urban street canyon. Science of the Total Environment 390, 437-447.

Kumar, P., Gurjar, B., Nagpure, A., Harrison, R.M., 2011a. Preliminary estimates of nanoparticle number emissions from road vehicles in megacity Delhi and associated health impacts. Environmental Science \& Technology $45,5514-5521$.

Kumar, P., Ketzel, M., Vardoulakis, S., Pirjola, L., Britter, R., 2011b. Dynamics and dispersion modelling of nanoparticles from road traffic in the urban atmospheric environment-A review. Journal of Aerosol Science 42, 580-603.

Kumar, P., Robins, A., Britter, R., 2009. Fast response measurements of the dispersion of nanoparticles in a vehicle wake and a street canyon. Atmospheric Environment 43, 6110-6118.

Kumar, P., Robins, A., Vardoulakis, S., Britter, R., 2010. A review of the characteristics of nanoparticles in the urban atmosphere and the prospects for developing regulatory controls. Atmospheric Environment 44, 50355052.

Kumar, P., Robins, A., Vardoulakis, S., Quincey, P., 2011c. Technical challenges in tackling regulatory concerns for urban atmospheric nanoparticles. Particuology 9, 566-571.

Lavine, M., Lozier, S., 1999. A Markov random field spatio-temporal analysis of ocean temperature. Environmental and Ecological Statistics 6, 249-273. 
Mishra, V.K., Kumar, P., Van Poppel, M., Bleux, N., Frijns, E., Reggente, M., Berghmans, P., Int Panis, L., Samson, R., 2012. Wintertime spatiotemporal variation of ultrafine particles in a Belgian city. Science of the Total Environment 431, 307-313.

Mølgaard, B., Hussein, T., Corander, J., Hmeri, K., 2012. Forecasting sizefractionated particle number concentrations in the urban atmosphere. Atmospheric Environment 46, 155-163.

Morawska, L., Ristovski, Z., Jayaratne, E., Keogh, D., Ling, X., 2008. Ambient nano and ultrafine particles from motor vehicle emissions: Characteristics, ambient processing and implications on human exposure. Atmospheric Environment 42, 8113-8138.

Morawska, L., Thomas, S., Bofinger, N., Wainwright, D., Neale, D., 1998. Comprehensive characterization of aerosols in a subtropical urban atmosphere: particle size distribution and correlation with gaseous pollutants. Atmospheric Environment 32, 2467-2478.

Morawska, L., Thomas, S., Gilbert, D., Greenaway, C., Rijnders, E., 1999. A study of the horizontal and vertical profile of submicrometer particles in relation to a busy road. Atmospheric Environment 33, 1261-1274.

Nikolova, I., Janssen, S., Vos, P., Vrancken, K., Mishra, V., Berghmans, P., 2011. Dispersion modelling of traffic induced ultrafine particles in a street canyon in Antwerp, Belgium and comparison with observations. Science of the Total Environment 412413, 336-343.

Octave community, GNU/Octave, 2014. www.gnu.org/software/octave. 
Pey, J., Querol, X., Alastuey, A., Rodrguez, S., Putaud, J.P., Dingenen, R.V., 2009. Source apportionment of urban fine and ultra-fine particle number concentration in a western mediterranean city. Atmospheric Environment 43, 4407-4415.

Rasmussen, C.E., 1996. Evaluation of Gaussian Processes and other Methods for Non-linear Regression. Ph.D. thesis. Department of Computer Science, University of Toronto.

Rasmussen, C.E., Ghahramani, Z., 2001. Occam's razor, in: In Advances in Neural Information Processing Systems 13, MIT Press. pp. 294-300.

Rasmussen, C.E., Ghahramani, Z., 2002. Infinite mixtures of Gaussian process experts, in: In Advances in Neural Information Processing Systems 14, MIT Press. pp. 881-888.

Rasmussen, C.E., Nickisch, H., 2010. Gaussian processes for machine learning (GPML) toolbox. Journal of Machine Learning Research 11, 30113015.

Rasmussen, C.E., Williams, C.K.I., 2006. Gaussian Processes for Machine Learning. MIT Press.

Sabaliauskas, K., Jeong, C.H., Yao, X., Jun, Y.S., Jadidian, P., Evans, G.J., 2012. Five-year roadside measurements of ultrafine particles in a major Canadian city. Atmospheric Environment 49, 245-256.

Sardar, S.B., Fine, P.M., Mayo, P.R., Sioutas, C., 2005. Size-fractionated measurements of ambient ultrafine particle chemical composition in Los Angeles using the NanoMOUDI. Environmental Science \& Technology $39,932-944$. 
Snelson, E., Ghahramani, Z., 2006. Sparse Gaussian processes using pseudoinputs, in: Advances in Neural Information Processing Systems 18, MIT press. pp. $1257-1264$.

Soltic, P., Weilenmann, M., 2003. $\mathrm{NO}_{2} / \mathrm{NO}$ emissions of gasoline passenger cars and light-duty trucks with Euro-2 emission standard. Atmospheric Environment 37, 5207-5216.

Spiegelhalter, D.J., Best, N.G., Carlin, B.P., van der Linde, A., 2002. Bayesian measures of model complexity and fit (with discussion). Journal of the Royal Statistical Society, Series B 64, 583-639(57).

The MathWorks Inc., 2014. www.mathworks.com.

Wehner, B., Uhrner, U., von Lwis, S., Zallinger, M., Wiedensohler, A., 2009. Aerosol number size distributions within the exhaust plume of a diesel and a gasoline passenger car under on-road conditions and determination of emission factors. Atmospheric Environment 43, 1235-1245.

Westerdahl, D., Fruin, S., Sax, T., Fine, P.M., Sioutas, C., 2005. Mobile platform measurements of ultrafine particles and associated pollutant concentrations on freeways and residential streets in Los Angeles. Atmospheric Environment 39, 3597-3610.

Westmoreland, E.J., Carslaw, N., Carslaw, D.C., Gillah, A., Bates, E., 2007. Analysis of air quality within a street canyon using statistical and dispersion modelling techniques. Atmospheric Environment 41, 9195-9205.

Zhu, Y., Hinds, W.C., Kim, S., Shen, S., Sioutas, C., 2002. Study of ultrafine particles near a major highway with heavy-duty diesel traffic. Atmospheric Environment 36, 4323-4335. 


\section{Appendix A. Supplementary Material}

Supplementary material of Section 3: Material

Table A.4: Summary statistics of UFP number concentrations, $\mathrm{NO}, \mathrm{NO}_{2}$, $\mathrm{CO}$ and $\mathrm{O}_{3}$ concentrations, measured at the three sites at half hour resolution.

\begin{tabular}{lllllllll}
\hline & Variable & Mean & Std. & Median & Min. & Max. & Q1 & Q3 \\
\hline \multirow{5}{*}{ Site 1 } & $\mathrm{UFP}\left(\# \mathrm{~cm}^{-3}\right)$ & 22810 & 12934 & 20628 & 1768 & 88004 & 13190 & 29316 \\
& $\mathrm{NO}\left(\mu \mathrm{g} \mathrm{m}^{-3}\right)$ & 57 & 69 & 36 & 0 & 571 & 14 & 70 \\
& $\mathrm{NO}_{2}\left(\mu \mathrm{g} \mathrm{m}^{-3}\right)$ & 56 & 26 & 54 & 5 & 150 & 36 & 73 \\
& $\mathrm{CO}\left(\mu \mathrm{g} \mathrm{m}^{-3}\right)$ & 435 & 220 & 376 & 84 & 1658 & 286 & 515 \\
& $\mathrm{O}_{3}\left(\mu \mathrm{g} \mathrm{m}^{-3}\right)$ & 32 & 21 & 32 & 1 & 88 & 14 & 49 \\
& & & & & & & & \\
\multirow{5}{*}{ Site 2 } & $\mathrm{UFP}\left(\# \mathrm{~cm}^{-3}\right)$ & 21586 & 11249 & 19278 & 2168 & 80355 & 13866 & 27486 \\
& $\mathrm{NO}\left(\mu \mathrm{g} \mathrm{m}^{-3}\right)$ & 78 & 80 & 53 & 1 & 716 & 29 & 97 \\
& $\mathrm{NO}\left(\mu \mathrm{g} \mathrm{m}^{-3}\right)$ & 72 & 30 & 70 & 11 & 170 & 50 & 89 \\
& $\mathrm{CO}\left(\mu \mathrm{g} \mathrm{m}^{-3}\right)$ & - & - & - & - & - & - & - \\
& $\mathrm{O}_{3}\left(\mu \mathrm{g} \mathrm{m}^{-3}\right)$ & 27 & 19 & 23 & 3 & 83 & 9 & 41 \\
& & & & & & & \\
Site 3 & $\mathrm{UFP}\left(\# \mathrm{~cm}^{-3}\right)$ & 23219 & 14129 & 19518 & 2528 & 87210 & 13703 & 28883 \\
& $\mathrm{NO}\left(\mu \mathrm{g} \mathrm{m}^{-3}\right)$ & 69 & 101 & 31 & 1 & 854 & 11 & 85 \\
& $\mathrm{NO}\left(\mu \mathrm{g} \mathrm{m}^{-3}\right)$ & 62 & 33 & 57 & 7 & 218 & 37 & 82 \\
& $\mathrm{CO}\left(\mu \mathrm{g} \mathrm{m}^{-3}\right)$ & 322 & 221 & 270 & 25 & 1606 & 164 & 403 \\
$\mathrm{O}_{3}\left(\mu \mathrm{g} \mathrm{m}^{-3}\right)$ & 30 & 21 & 26 & 1 & 91 & 10 & 47 \\
\hline
\end{tabular}

Table A.4 provides the summary statistics for UFP, $\mathrm{NO}, \mathrm{NO}_{2}, \mathrm{CO}$ and $\mathrm{O}_{3}$ concentrations recorded over the entire sampling period at half hour resolution. 
Supplementary material of Section 4: Description of the model

Product of two Gaussians

The product of two Gaussian gives another Gaussian:

$$
\mathcal{N}(\mathbf{x} \mid \mathbf{a}, A) \mathcal{N}(\mathbf{x} \mid \mathbf{b}, B)=Z^{-1} \mathcal{N}(\mathbf{x} \mid \mathbf{c}, C)
$$

where

$$
\begin{gathered}
\mathbf{c}=C\left(A^{-1} \mathbf{a}+B^{-1} \mathbf{b}\right), \\
C=\left(A^{-1}+B^{-1}\right) \\
Z^{-1}=(2 \pi)^{D / 2}|A+B|^{-1 / 2} \exp \left(-\frac{1}{2}(\mathbf{a}-\mathbf{b})^{\top}(A+B)^{-1}(\mathbf{a}-\mathbf{b})\right) .
\end{gathered}
$$

The resulting Gaussian has the precision equal to the sum of the precisions, and the mean equal to the convex sum of the means weighted by the precisions. $Z$ is a normalizing constant.

Conditioning a joint Gaussian distribution

The formula for conditioning a joint Gaussian distribution is:

$$
\begin{aligned}
{\left[\begin{array}{l}
\mathbf{x} \\
\mathbf{y}
\end{array}\right] \sim \mathcal{N}\left(\left[\begin{array}{l}
\mathbf{a} \\
\mathbf{b}
\end{array}\right],\left[\begin{array}{cc}
A & C \\
C^{\top} & B
\end{array}\right]\right) \longrightarrow } \\
\longrightarrow \mathbf{x} \mid \mathbf{y} \sim \mathcal{N}\left(a+C B^{-1}(\mathbf{y}-b), A-C B^{-1} C^{\top}\right)
\end{aligned}
$$

Supplementary material of Section 6: Results and discussion

In Figure A.10 we show that the full GP models and FITC approximations have a similar performance for all the training lengths. The FITC approximations use $10 \%$ of the training data as inducing points. The models are based on half hour resolution data. 


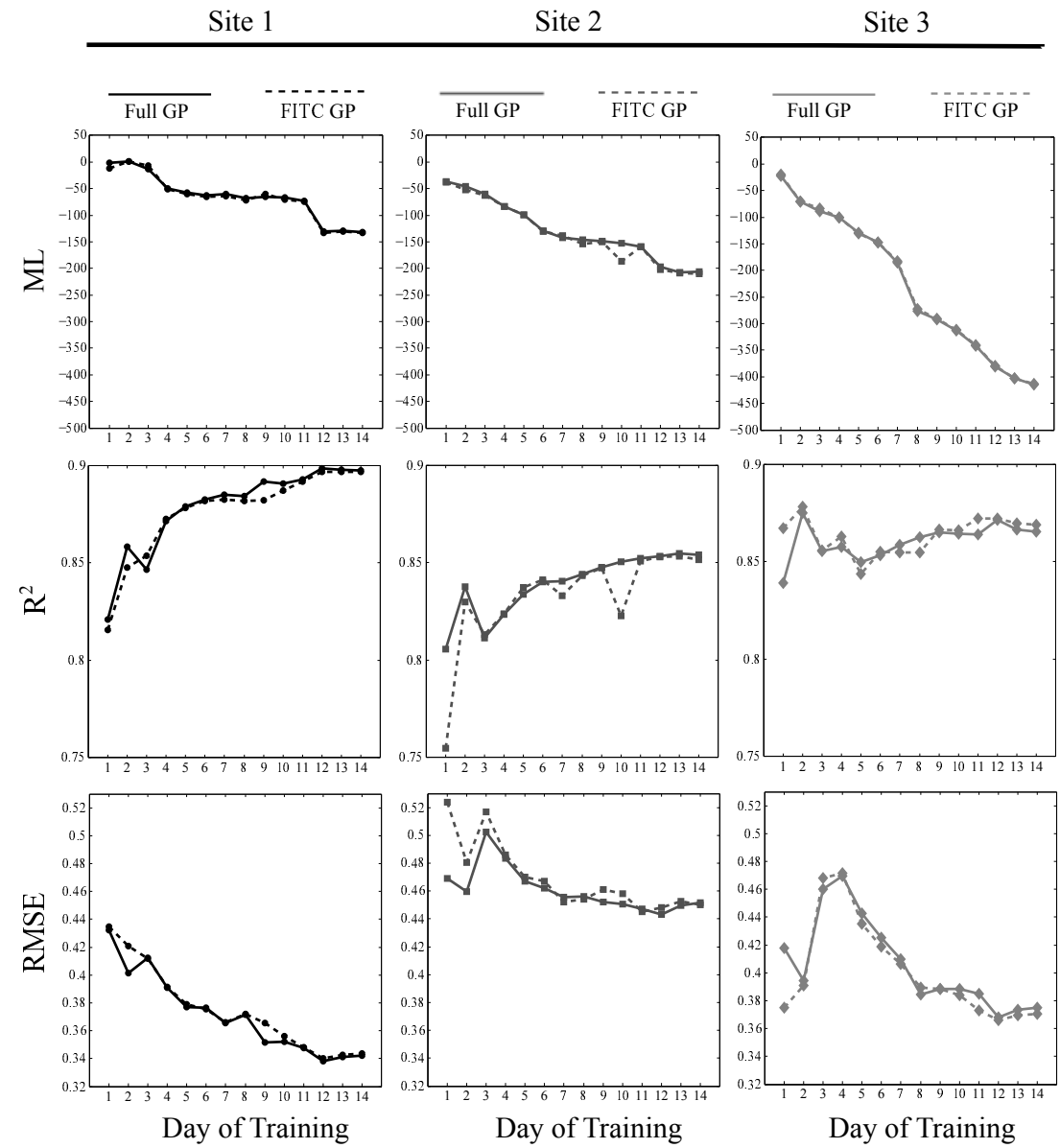

Figure A.10: Comparison between full GP regression (solid lines) and FITC approximation (dashed lines). 

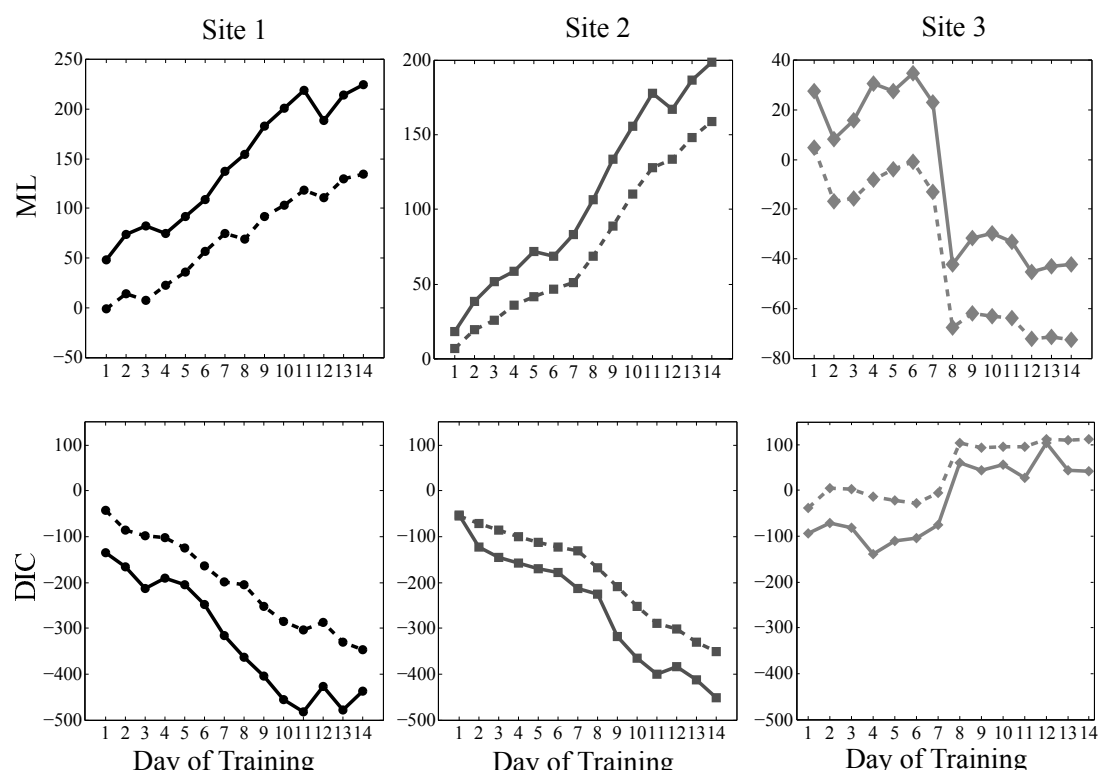

Figure A.11: Model evaluation (ML and DIC) for log-transformed data. Solid lines refer to GP regression, and dashed lines refer to Bayesian linear regression. 

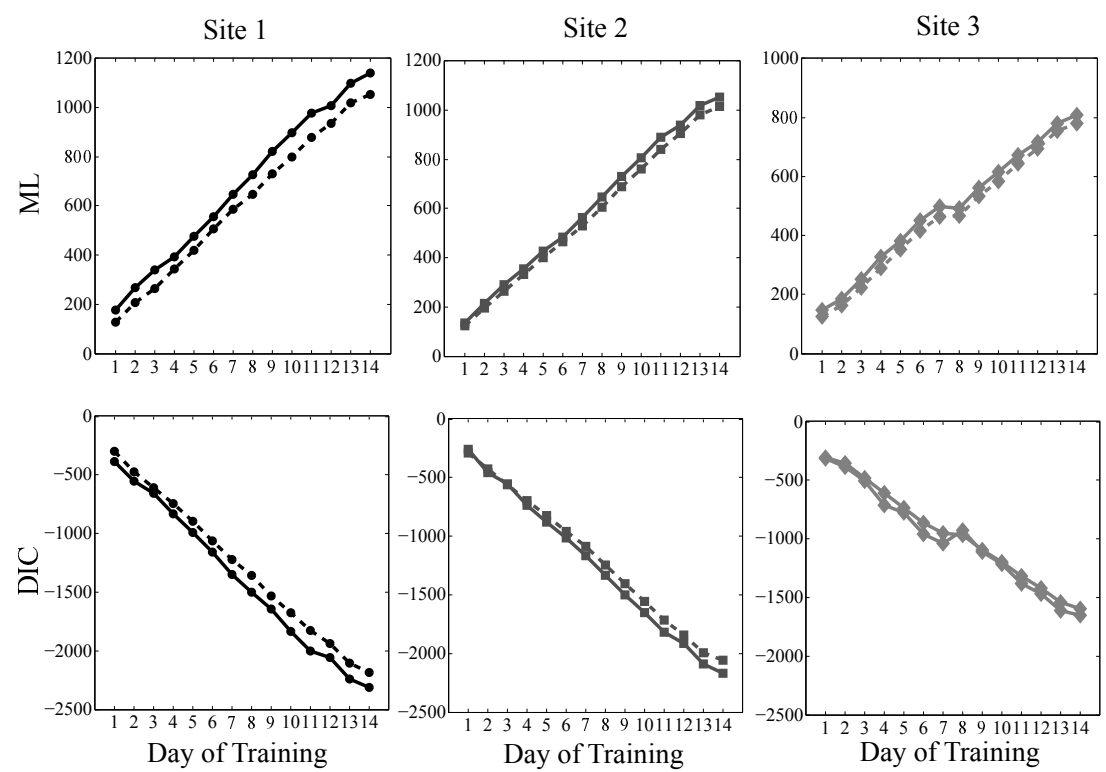

Figure A.12: Model evaluation (ML and DIC) for log-transformed data and zero to one scaled data. Solid lines refer to GP regression, and dashed lines refer to Bayesian linear regression. 
Comparing the ML and DIC trends in Figure 8 - in which the data is logtransformed and standardized with a zero mean and unit standard deviation - with the relative trends in Figure A.11 - in which the data is only logtransformed - and Figure A.12 - in which the data is log-transformed and scaled between zero and one - we can note that: (i) in the case of Figure 8 the ML and DIC values get worse when increasing the amount of training data, suggesting that the models that use only one day of training have the best increase in model fit with the lower model complexity; (ii) in the case of Figures A.11 and A.12 there are opposite trends and the ML and DIC values improve when increasing the amount of training data, suggesting that the models that uses longer periods are preferable.

Figure A.13 shows the posterior correlation matrix between the predicted UFP number concentrations and the inputs $\left(\mathrm{NO}\right.$ and $\left.\mathrm{NO}_{2}\right)$. At all sites, the covariate that is most related to the prediction (most informative) is $\mathrm{NO}_{2}$. 


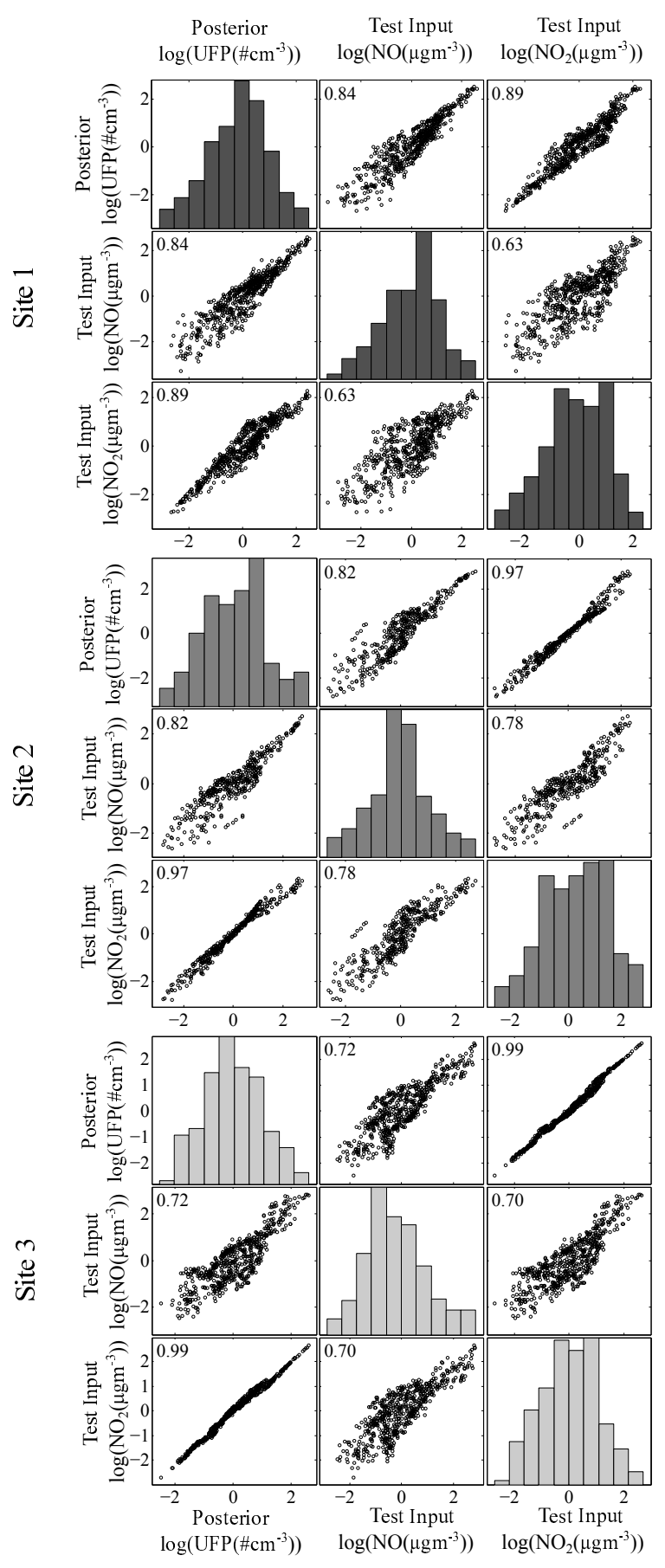

Figure A.13: Correlation matrix between the test inputs $\left(\mathrm{NO}\right.$ and $\left.\mathrm{NO}_{2}\right)$ and the predicted UFP number concentrations (posterior). 